\title{
THE ELM SURVEY. IV. 24 WHITE DWARF MERGER SYSTEMS*
}

\author{
Mukremin Kilic ${ }^{1}$, Warren R. Brown ${ }^{2}$, Carlos Allende Prieto ${ }^{3,4}$, S. J. Kenyon ${ }^{2}$, Craig O. Heinke ${ }^{5}$, \\ M. A. AgüEros ${ }^{6}$, and S. J. Kleinman ${ }^{7}$ \\ ${ }^{1}$ Homer L. Dodge Department of Physics and Astronomy, University of Oklahoma, 440 West Brooks Street, Norman, OK 73019, USA; kilic@ ou.edu \\ ${ }^{2}$ Smithsonian Astrophysical Observatory, 60 Garden Street, Cambridge, MA 02138, USA \\ ${ }^{3}$ Instituto de Astrofísica de Canarias, E-38205 La Laguna, Tenerife, Spain \\ ${ }^{4}$ Departamento de Astrofísica, Universidad de La Laguna, E-38206 La Laguna, Tenerife, Spain \\ ${ }^{5}$ Department of Physics, CCIS 4-183, University of Alberta, Edmonton, AB, T6G 2E1, Canada \\ ${ }^{6}$ Department of Astronomy, Columbia University, 550 West 120th Street, New York, NY 10027, USA \\ ${ }^{7}$ Gemini Observatory, 670 North A'ohoku Place, Hilo, HI 96720, USA \\ Received 2012 January 5; accepted 2012 March 30; published 2012 May 16
}

\begin{abstract}
We present new radial velocity and X-ray observations of extremely low mass (ELM, $\sim 0.2 M_{\odot}$ ) white dwarf (WD) candidates in the Sloan Digital Sky Survey Data Release 7 area. We identify seven new binary systems with 1-18 hr orbital periods. Five of the systems will merge due to gravitational wave radiation within 10 Gyr, bringing the total number of merger systems found in the ELM Survey to 24. The ELM Survey has now quintupled the known merger WD population. It has also discovered the eight shortest period detached binary WD systems currently known. We discuss the characteristics of the merger and non-merger systems observed in the ELM Survey, including their future evolution. About half of the systems have extreme mass ratios. These are the progenitors of the AM Canum Venaticorum systems and Type Ia supernovae. The remaining targets will lead to the formation of extreme helium stars, subdwarfs, or massive WDs. We identify three targets that are excellent gravitational wave sources. These should be detected by the Laser Interferometer Space Antenna like missions within the first year of operation. The remaining targets are important indicators of what the Galactic foreground may look like for gravitational wave observatories.
\end{abstract}

Key words: binaries: close - Galaxy: stellar content - gravitational waves - supernovae: general - white dwarfs

Online-only material: color figures

\section{INTRODUCTION}

Short-period binary white dwarfs (WDs) are strong gravitational wave sources and the potential progenitors of Type Ia (Webbink 1984; Iben \& Tutukov 1984) and .Ia supernovae (Bildsten et al. 2007). The gravitational wave radiation and the orbital decay in the shortest period systems may be detected directly by space-based missions like the Laser Interferometer Space Antenna (LISA) and indirectly by ground-based observations (see Brown et al. 2011c). Nelemans (2009) lists 12 ultracompact systems that are guaranteed LISA sources, but predicts that LISA should detect at least several hundred systems.

The extremely low mass (ELM) Survey (Kilic et al. 2010b, 2011a; Brown et al. 2010, 2012) is opening a new window on short-period binary WDs and strong gravitational wave sources. After the discovery of four double WD systems with merger times shorter than 500 Myr (Kilic et al. 2010b), radial velocity follow-up of the ELM WDs found in the Hypervelocity star survey (Brown et al. 2006) and the Sloan Digital Sky Survey (SDSS) Data Release 4 sample (Eisenstein et al. 2006) led to the discovery of 12 merger systems, tripling the number of known merging WD systems (Brown et al. 2010; Kilic et al. 2011a). In 2011, the ELM Survey identified the three shortest period detached binary WDs known, a 12 minute orbital period eclipsing system (Brown et al. 2011c) and two 39 minute orbital period systems (Kilic et al. 2011b, 2011c). All three systems show flux variations due to the relativistic beaming effect and two of the three also show ellipsoidal variations due to tidal distortions. These are the first two tidally distorted WDs ever

\footnotetext{
* Based on observations obtained at the MMT Observatory, a joint facility of the Smithsonian Institution and the University of Arizona.
}

found. The three systems with $<1 \mathrm{hr}$ orbital periods are strong gravitational wave sources.

Marsh et al. (1995) first demonstrated that the majority of lowmass $\left(\leqslant 0.45 M_{\odot}\right)$ WDs are found in binaries, as the Galaxy is too young to form such objects from single stars. Brown et al. (2011b) show that the binary fraction of low-mass WDs is at least $70 \%$. This fraction goes up to $100 \%$ for ELM WDs with $M<0.25 M_{\odot}$ (Kilic et al. 2011a). The Supernovae Progenitor Survey (Napiwotzki et al. 2001), on the other hand, finds a significantly lower binary fraction for typical $0.6 M_{\odot}$ WDs and only a handful of binaries that will merge within a Hubble time (Napiwotzki et al. 2007). Hence, ELM WDs provide the best opportunity to study the population of short-period binary WDs.

In paper I (Brown et al. 2010) of this series, we studied the population of ELM WDs found in the Hypervelocity star survey. In paper II (Kilic et al. 2011a), we presented the SDSS Data Release 4 systems. In paper III (Brown et al. 2012), we performed a targeted spectroscopic survey of cooler ( $\simeq 10,000 \mathrm{~K})$ ELM WDs selected by color. Here, we extend our survey to the SDSS Data Release 7 sample.

Section 2 describes our target selection, radial velocity, and X-ray observations. Section 3 presents the orbital and physical parameters of the seven binaries that we targeted for spectroscopic observations. The entire population of 40 systems observed in the ELM Survey to date is presented in Section 4 along with a discussion of the most interesting systems and trends. Section 5 lists our conclusions and future prospects.

\section{OBSERVATIONS}

\subsection{Optical Spectroscopy}

Kleinman (2010) identifies 12 new ELM WD candidates in the SDSS Data Release 7 spectroscopy data, including 
Table 1

X-Ray Observations of ELM WDs

\begin{tabular}{|c|c|c|c|c|c|c|}
\hline Name & ObsID & $\begin{array}{c}\text { Distance } \\
\text { (pc) }\end{array}$ & $\begin{array}{c}N_{\mathrm{H}} \\
\left(\mathrm{cm}^{-2}\right)\end{array}$ & $\begin{array}{c}\text { Exposure } \\
(\mathrm{ks})\end{array}$ & $\begin{array}{l}\text { Count Rate } \\
\quad\left(\text { cts s}^{-1}\right)\end{array}$ & $\begin{array}{c}L_{X} \\
\left(\operatorname{erg~s}^{-1}\right)\end{array}$ \\
\hline SDSS J082212.57+275307.4 & 12352 & 430 & $3.5 \times 10^{20}$ & 2.0 & $<2.2 \times 10^{-3}$ & $<2.2 \times 10^{29}$ \\
\hline SDSS J084910.13+044528.7 & 12354 & 930 & $4.1 \times 10^{20}$ & 10.9 & $<4.2 \times 10^{-4}$ & $<2.0 \times 10^{29}$ \\
\hline
\end{tabular}

Notes. 99\% confidence X-ray count rate upper limits for two ELM WDs from Chandra X-ray observations. Count rate limit is in the 0.3-6 keV band and $L_{X}$ limit is in the $0.5-6 \mathrm{keV}$ band.

J0106-1000 (Kilic et al. 2011c), J0923+3028 (Brown et al. 2010), J1518+0658 (Brown et al. 2012), and J0651+2844 (Brown et al. 2011c). Here we focus on the remaining eight objects from this sample, plus J1056+6536 from the SDSS Data Release 4 WD catalog (Eisenstein et al. 2006). We present observations of seven targets with reliable orbital solutions. The remaining targets need more observations to constrain their orbital parameters and they will be discussed in a future paper.

We used the $6.5 \mathrm{~m}$ MMT equipped with the Blue Channel spectrograph over several different observing runs between 2010 March and 2011 October. We operate the spectrograph with the 832 line $\mathrm{mm}^{-1}$ grating in second order, providing wavelength coverage $3650-4500 \AA$ and a spectral resolution of $1.2 \AA$. All objects were observed at the parallactic angle, and a comparison lamp exposure was obtained with every observation. We flux-calibrate using blue spectrophotometric standards (Massey et al. 1988).

We measure radial velocities using the cross-correlation package RVSAO (Kurtz \& Mink 1998). We first cross-correlate the observed spectra with a high signal-to-noise WD template. We then shift the observed spectra to the rest frame, and sum them together to create a template for each object. Finally, we cross-correlate the spectra with the appropriate template to obtain the final velocities for each object. The average precision of our measurements is $20 \mathrm{~km} \mathrm{~s}^{-1}$.

We compute best-fit orbital elements using the code of Kenyon \& Garcia (1986), which weights each velocity measurement by its associated error. We perform a Monte Carlo analysis to verify the uncertainties in the orbital parameters (see Brown et al. 2012).

\subsection{X-Ray Observations}

\subsubsection{Motivation}

The probability of neutron star companions to the majority of the objects identified in the ELM Survey is only a few percent. However, based on the mass function, there are several ELM WD systems where the probability of a neutron star (which would be spun up to a millisecond pulsar (MSP)) companion is more than $10 \%$. Radio and X-ray observations are essential to confirm or rule out such companions.

Agüeros et al. (2009b) and Kilic et al. (2011a) discuss the importance of X-ray observations for the identification of MSP companions to ELM WDs. Blackbody emission from the surface of a possible pulsar companion to the ELM WDs will be gravitationally bent, allowing observation of $>75 \%$ of the neutron star surface in X-rays even if the radio pulsar beam misses our line of sight (Beloborodov 2002). All 15 radio MSPs with precise positions in unconfused regions of the globular cluster 47 Tuc have been clearly detected in X-rays (Heinke et al. 2005; Bogdanov et al. 2006). This result allows us to use the 47 Tuc MSP sample (with accurate $\mathrm{X}$-ray luminosities, $L_{X}$, due to its well-known distance) to predict that other MSPs should have X-ray luminosities above $L_{X}(0.5-6 \mathrm{keV})=2 \times 10^{30} \mathrm{erg} \mathrm{s}^{-1}$, the minimum $L_{X}$ of MSPs in 47 Tuc. Thus, deep X-ray observations can confirm or rule out the presence of MSP companions in the ELM WD binary systems.

We obtained Chandra observations of two previously known ELM WDs, J082212.57+275307.4 and J084910.13+044528.7 (Kilic et al. 2010b), to search for X-ray emission from an MSP. Based on the mass function, there is a $15 \%-18 \%$ probability that these stars have neutron star (1.4-3 $\left.M_{\odot}\right)$ companions. Neither had been previously observed in X-rays since the ROSAT AllSky Survey (Voges et al. 1999), which neither detected them nor placed useful limits.

\subsubsection{Data Analysis}

We used Chandra's ACIS-S detector in Very Faint mode to observe J082212.57+275307.4 for 2.0 ks on 2011 December 13, and J084910.13+044528.7 for $10.9 \mathrm{ks}$ on 2011 March 2 (Table 1). We used CIAO $4.3^{8}$ and CALDB 4.4.2 to reprocess the data including current calibrations, reducing the backgrounds using Very Faint mode cleaning. We constructed images in the $0.3-6 \mathrm{keV}$ band, and found no X-ray photons within the $1^{\prime \prime}$ error circles around each source. We compute distances to the ELM WDs using the models of Panei et al. (2007) and the SDSS photometry, and the neutral hydrogen column density $N_{\mathrm{H}}$ using the Colden tool ${ }^{9}$ (Dickey \& Lockman 1990). We use PIMMS ${ }^{10}$ and the X-ray spectrum of the faintest MSP in 47 Tuc (47 Tuc-T, 134 eV blackbody) to produce $0.5-6 \mathrm{keV} L_{X}$ upper limits, which we list in Table 1.

The $99 \%$ confidence upper limits we calculate are an order of magnitude lower than the faintest MSP observed in 47 Tuc, and factors of 19 and 20 fainter than the median $L_{X}$ of the 47 Tuc MSPs. Therefore, the lack of detected X-ray emission from these two WDs is strong evidence that their companions are not MSPs.

\section{RESULTS}

Our seven targets with optical spectroscopy data were classified as ELM WDs based on lower resolution and signal-tonoise ratio SDSS spectra. We use the MMT spectra to improve the model atmosphere analysis for these targets. We perform stellar atmosphere model fits using synthetic DA WD spectra kindly provided by D. Koester and an evolved version of the analysis code presented by Allende Prieto et al. (2006). We fit the flux-calibrated spectra as well as the continuum-corrected Balmer line profiles. Using the spectral continuum provides improved constraints on effective temperature. We compare bestfit solutions and find that the parameters differ on average by

\footnotetext{
8 http://cxc.cfa.harvard.edu/ciao/

9 http://asc.harvard.edu/toolkit/colden.jsp

$10 \mathrm{http}: / /$ asc.harvard.edu/toolkit/pimms.jsp
} 

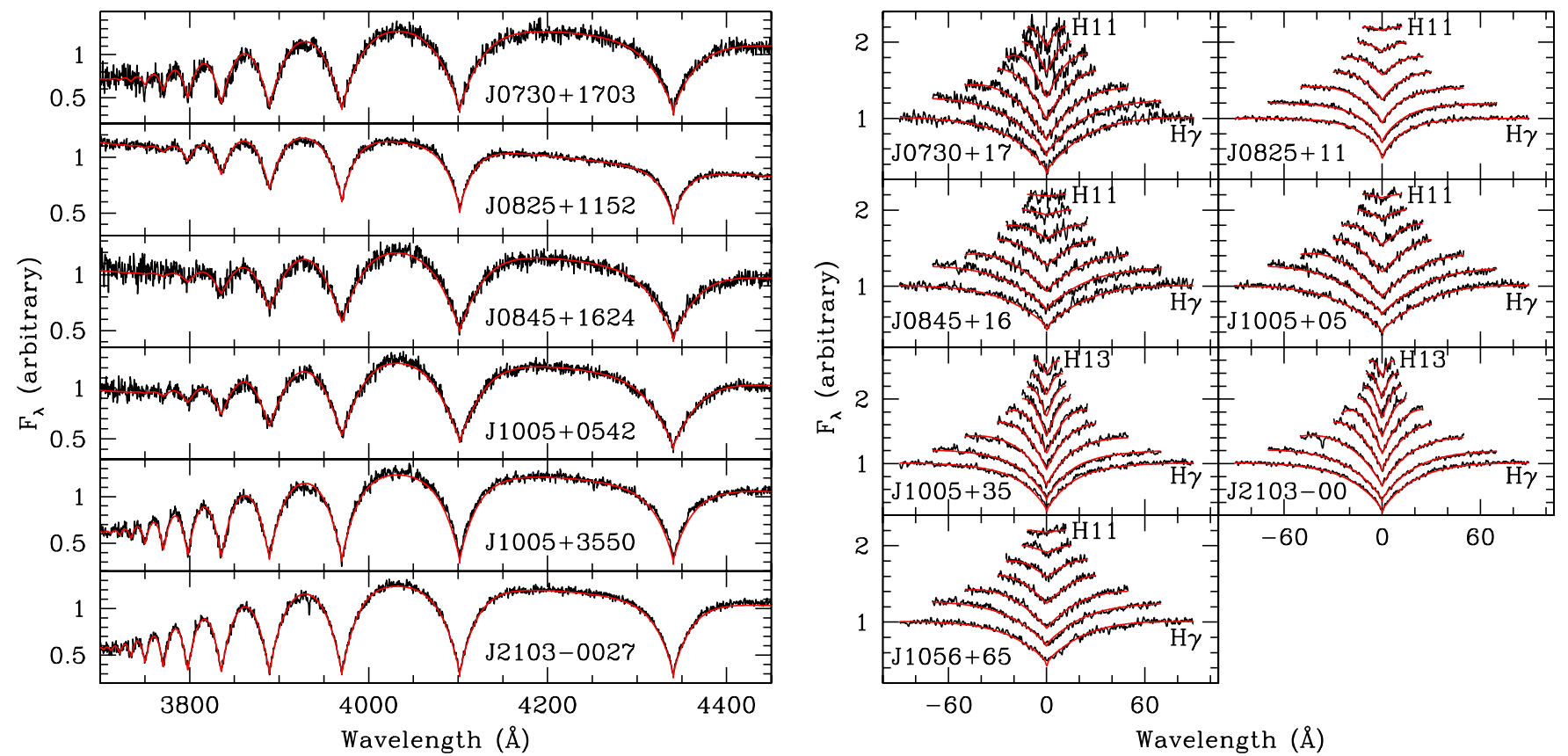

Figure 1. Spectral fits (red solid lines) to the composite spectra of our targets (jagged lines, left panels) and to the flux-normalized line profiles (right panels). The composite spectrum of J1056+6536 suffers from flux calibration problems and is not shown in the left panels.

(A color version of this figure is available in the online journal.)

$380 \pm 570 \mathrm{~K}$ in $T_{\text {eff }}$ and $0.03 \pm 0.05$ dex in $\log g$. We take these differences as our systematic errors. We have 14-23 individual spectra for each object. We use the fits to the individual spectra to derive a robust statistical error estimate. Eisenstein et al. (2006) and Kleinman (2010) use the SDSS spectra to derive physical parameters for the same targets. Our parameters differ on average by $30 \pm 520 \mathrm{~K}$ in $T_{\text {eff }}$ and $0.17 \pm 0.08$ dex in $\log g$.

Figure 1 shows the composite spectra and the best-fit models for our targets. These models provide a good match to the observed composite spectra and the Balmer line profiles. The parameters from both the flux-calibrated and continuum-corrected fits are in good agreement with the SDSS photometry in all five filters, an indication that our temperature and surface gravity measurements are reliable. We detect flux calibration problems for only one of our targets, J1056+6536, where the model using the continuum shape is not a good match to the observations. We use the results from the Balmer line profile fitting for this star. This model agrees remarkably well with the spectral energy distribution based on the SDSS photometry.

Figure 2 compares the best-fit $T_{\text {eff }}$ and $\log g$ measurements for our targets against the predicted evolutionary sequences from Panei et al. (2007). Based on these tracks, our targets have $M=0.17-0.40 M_{\odot}$; some of them are more massive than predicted from the relatively noisy SDSS spectroscopy data. The physical parameters of all seven systems discussed in this paper are presented in Table 2 . The age and distance estimates are somewhat uncertain for $M \approx 0.17 M_{\odot}$ objects, because many of them fall in the gap between 0.17 and $0.18 M_{\odot}$ He-core WD tracks. Panei et al. (2007) and Kilic et al. (2010b) argue that diffusion-induced hydrogen-shell flashes take place for $M>0.17 M_{\odot}$, which yield small hydrogen envelopes. Hence, lower mass objects have massive hydrogen envelopes, larger radii, lower surface gravities, and longer cooling times. The inconsistency between the observed parameters for $T_{\text {eff }} \sim$ $10,000 \mathrm{~K}$ and $\log g \leqslant 6$ objects and the Panei et al. (2007, Figure 2) models makes accurate WD mass and luminosity

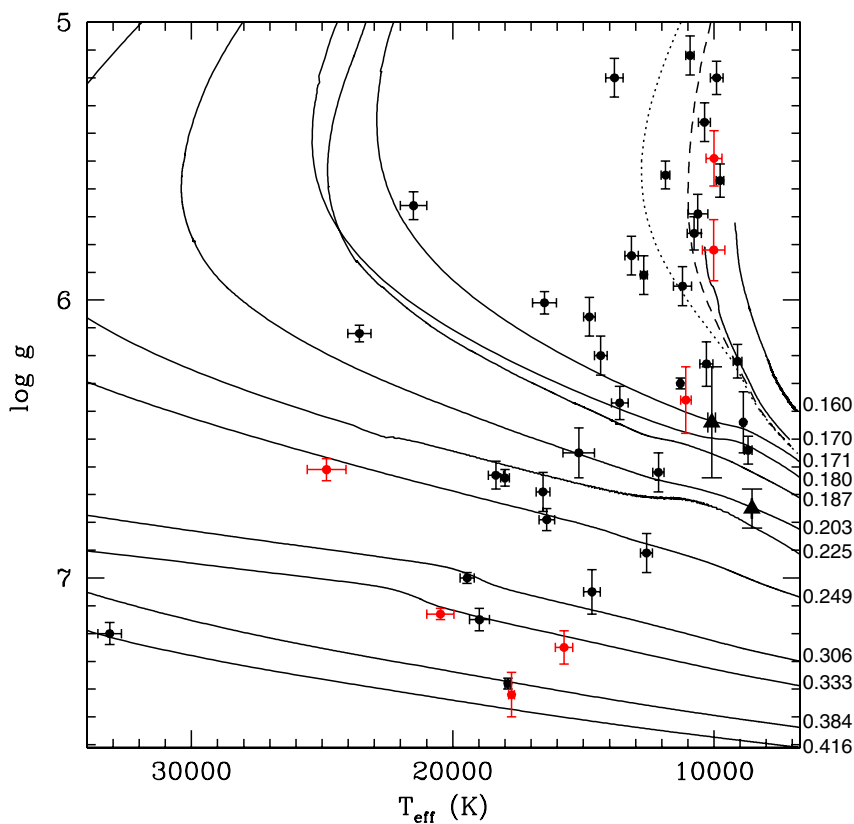

Figure 2. Surface gravity vs. effective temperature of the observed WDs (filled points) in the ELM Survey, compared with predicted tracks for He WDs with $0.16-0.42 M_{\odot}$ (Panei et al. 2007). The dashed and dotted lines show solar metallicity and halo metallicity $(Z=0.001)$ models of Serenelli et al. (2001, 2002) for $0.17 M_{\odot}$ WDs, respectively. The seven new systems presented in this paper are shown as red points. Triangles show the ELM WD companions to PSR J1012+5307 and J1911-5958A.

(A color version of this figure is available in the online journal.)

estimates difficult for them. Fortunately, mass and luminosity change very little over the range of effective temperature and surface gravity sampled by these WDs. We adopt $M=0.17 M_{\odot}$ and $M_{g} \simeq 8$ mag for these objects.

All seven targets show significant velocity variations with peak-to-peak velocity amplitudes of $120-640 \mathrm{~km} \mathrm{~s}^{-1}$ and 

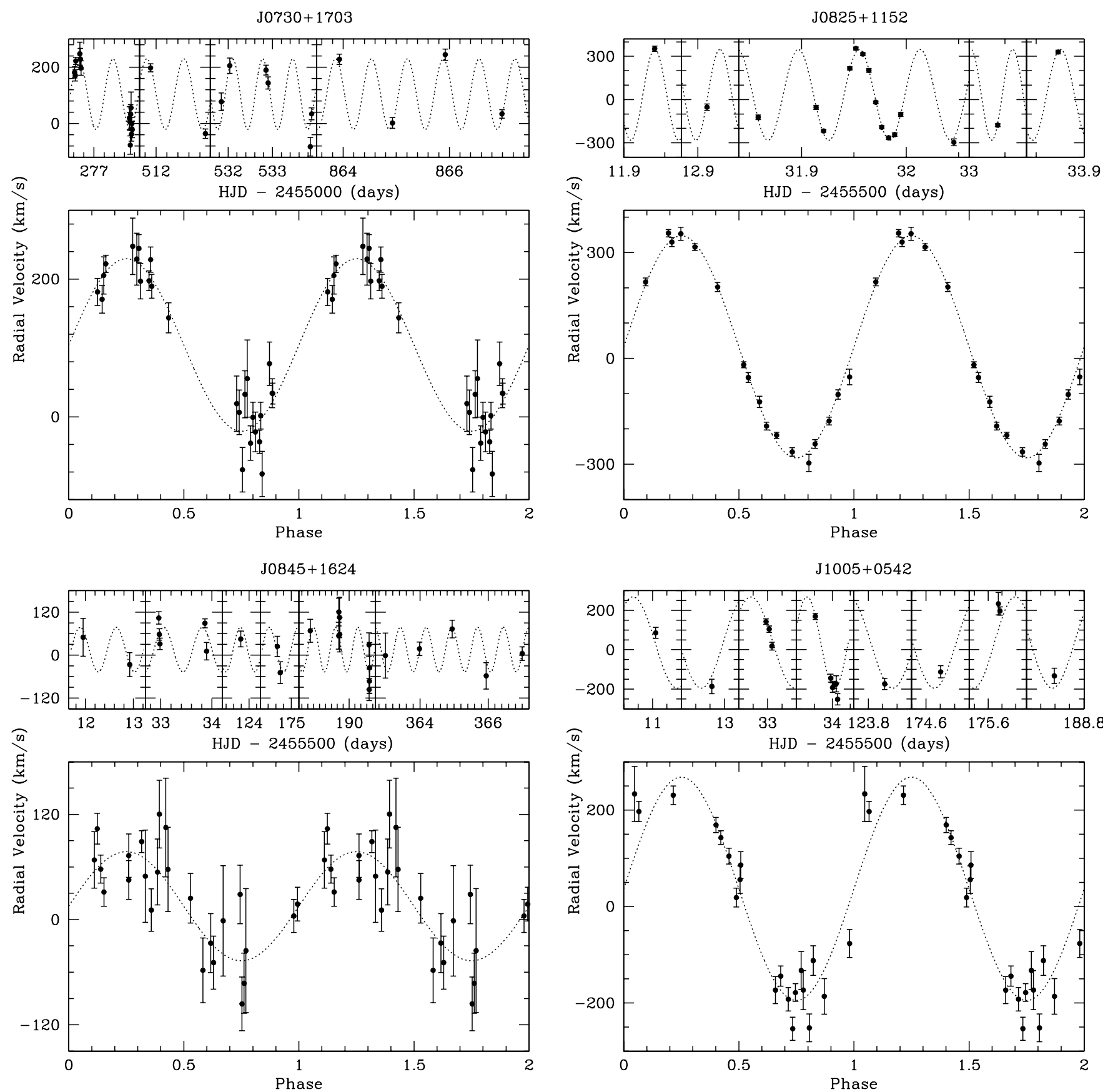

Figure 3. Velocities and orbits for the seven new ELM WD candidates. Small panels plot the heliocentric radial velocities vs. observation date. Large panels plot the observations phased to the best-fit orbital solutions (Table 3).

1-18 hr orbital periods. Figure 3 shows the observed radial velocities and the best-fit orbits for our targets. We present the best-fit orbital period $(P)$, semi-amplitude $(K)$ of the radial velocity variations, systemic velocity $(\gamma$, which includes a small gravitational redshift term), the time of spectroscopic conjunction (the time when the primary is closest to us), and the mass function in Table 3. The correction for the gravitational redshift is a couple of $\mathrm{km} \mathrm{s}^{-1}$ for a $0.17 M_{\odot}$ helium WD, comparable to the systemic velocity uncertainty. Based on the mass function alone, the companions would range from $M \geqslant 0.19 M_{\odot}$ (for $\mathrm{J} 0845+1624$ and $\mathrm{J} 1005+3550$ ) to $M \geqslant 0.71 M_{\odot}$ objects (for J2103-0027). Such main-sequence companions would be detected in the SDSS photometry and spectroscopy (Kroupa \& Tout 1997). Hence, the companions are either more massive WDs or neutron stars (MSPs). We discuss each binary in turn.

\section{1. $J 0730+1703$}

The ELM WD J0730+1703 has $T_{\text {eff }}=11,080 \pm 200 \mathrm{~K}$ and $\log g=6.36 \pm 0.12$. It is a $1.1 \mathrm{Gyr}$ old $0.17 \pm 0.01 M_{\odot}$ WD at a distance of $1.2 \mathrm{kpc}$. It has a large systemic velocity of $103 \mathrm{~km} \mathrm{~s}^{-1}$ but a relatively small proper motion of $2.8 \pm$

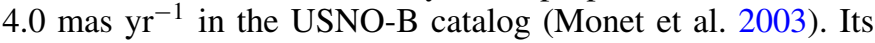
kinematics are consistent with the disk population.

$\mathrm{J} 0730+1703$ has a best-fit orbital period of $16.7 \mathrm{hr}$, but the current data set allows for a significant alias at $9.9 \mathrm{hr}$. The relatively small $122.8 \mathrm{~km} \mathrm{~s}^{-1}$ radial velocity semi-amplitude of 

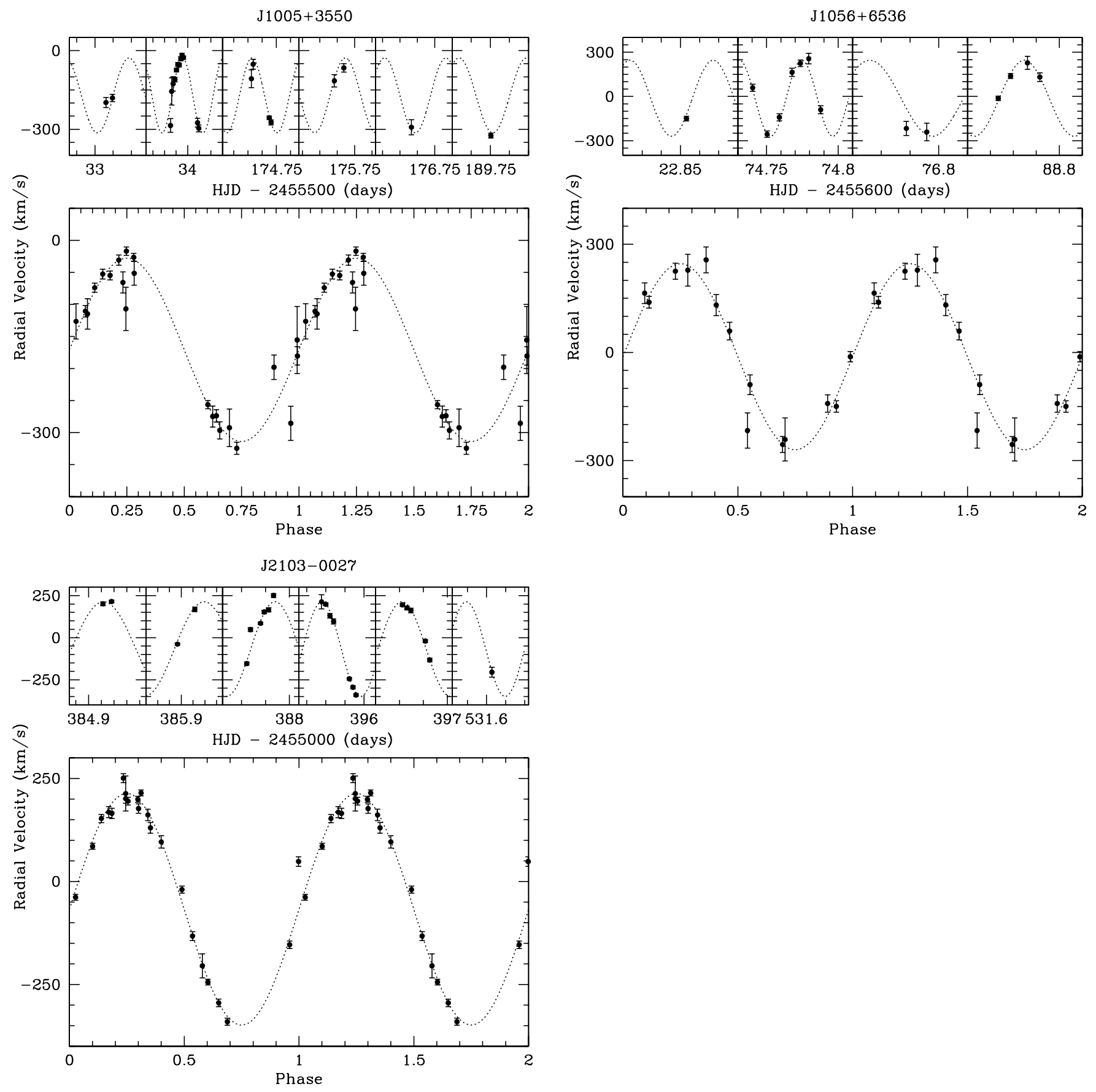

Figure 3. (Continued)

Table 2

Physical Parameters

\begin{tabular}{|c|c|c|c|c|c|c|c|c|c|c|}
\hline Object & $\begin{array}{c}g_{0} \\
(\mathrm{mag})\end{array}$ & $\begin{array}{l}T_{\text {eff }} \\
(\mathrm{K})\end{array}$ & $\begin{array}{c}\log g \\
\left(\mathrm{~cm} \mathrm{~s}^{-1}\right)\end{array}$ & $\begin{array}{l}\text { Mass } \\
\left(M_{\odot}\right) \\
\end{array}$ & $\begin{array}{c}M_{2} \\
\left(M_{\odot}\right)\end{array}$ & $\begin{array}{c}M_{2}\left(60^{\circ}\right) \\
\left(M_{\odot}\right)\end{array}$ & $\begin{array}{c}d \\
(\mathrm{kpc})\end{array}$ & $\begin{array}{l}\text { NS } \\
\text { Prob. }\end{array}$ & $\begin{array}{l}\text { SN Ia } \\
\text { Prob. }\end{array}$ & $\begin{array}{l}\tau_{\text {merge }} \\
(\mathrm{Gyr})\end{array}$ \\
\hline J0730+1703 & 19.7 & $11080 \pm 200$ & $6.36 \pm 0.12$ & 0.17 & $\geqslant 0.32$ & 0.41 & 1.2 & $6 \%$ & $2 \%$ & $\leqslant 266$ \\
\hline J0825+1152 & 18.6 & $24830 \pm 740$ & $6.61 \pm 0.04$ & 0.26 & $\geqslant 0.47$ & 0.61 & 1.6 & $9 \%$ & $4 \%$ & $\leqslant 0.180$ \\
\hline J0845+1624 & 19.7 & $17750 \pm 110$ & $7.42 \pm 0.08$ & 0.40 & $\geqslant 0.19$ & 0.22 & 0.9 & $2 \%$ & $2 \%$ & $\leqslant 251$ \\
\hline $\mathrm{J} 1005+0542$ & 19.7 & $15740 \pm 330$ & $7.25 \pm 0.06$ & 0.34 & $\geqslant 0.66$ & 0.86 & 1.0 & $14 \%$ & $11 \%$ & $\leqslant 9.0$ \\
\hline $\mathrm{J} 1005+3550$ & 18.8 & $10010 \pm 430$ & $5.82 \pm 0.11$ & 0.17 & $\geqslant 0.19$ & 0.24 & 1.5 & $3 \%$ & $1 \%$ & $\leqslant 10.3$ \\
\hline J1056+6536 & 19.7 & $20470 \pm 520$ & $7.13 \pm 0.02$ & 0.34 & $\geqslant 0.34$ & 0.43 & 1.4 & $5 \%$ & $4 \%$ & $\leqslant 0.085$ \\
\hline J2103-0027 & 18.2 & $10000 \pm 300$ & $5.49 \pm 0.10$ & 0.17 & $\geqslant 0.71$ & 0.99 & 1.1 & $17 \%$ & $5 \%$ & $\leqslant 5.4$ \\
\hline
\end{tabular}


Table 3

Orbital Parameters

\begin{tabular}{|c|c|c|c|c|c|}
\hline Object & $\begin{array}{c}P \\
\text { (days) }\end{array}$ & $\begin{array}{c}K \\
\left(\mathrm{~km} \mathrm{~s}^{-1}\right)\end{array}$ & $\begin{array}{c}\gamma \\
\left(\mathrm{km} \mathrm{s}^{-1}\right)\end{array}$ & $\begin{array}{l}\text { Spec. Conjunction } \\
\text { (HJD-2455000) }\end{array}$ & $\begin{array}{l}\text { Mass Function } \\
\qquad\left(M_{\odot}\right)\end{array}$ \\
\hline J073032.89+170356.9 & $0.69770 \pm 0.05427$ & $122.8 \pm 4.3$ & $103.1 \pm 3.4$ & $276.53156 \pm 0.00612$ & $0.1339 \pm 0.0175$ \\
\hline J082511.90+115236.4 & $0.05819 \pm 0.00001$ & $319.4 \pm 2.7$ & $32.7 \pm 2.9$ & $511.92318 \pm 0.00146$ & $0.1964 \pm 0.0050$ \\
\hline $\mathrm{J} 084523.03+162457.6$ & $0.75599 \pm 0.02164$ & $62.2 \pm 5.4$ & $15.2 \pm 4.6$ & $511.69953 \pm 0.01308$ & $0.0188 \pm 0.0049$ \\
\hline J100548.09+054204.4 & $0.30560 \pm 0.00007$ & $208.9 \pm 6.8$ & $8.6 \pm 8.2$ & $510.87236 \pm 0.00359$ & $0.2886 \pm 0.0282$ \\
\hline $\mathrm{J} 100554.05+355014.2$ & $0.17652 \pm 0.00011$ & $143.0 \pm 2.3$ & $-170.9 \pm 1.7$ & $533.04951 \pm 0.00058$ & $0.0535 \pm 0.0026$ \\
\hline $\mathrm{J} 105611.02+653631.5$ & $0.04351 \pm 0.00103$ & $267.5 \pm 7.4$ & $-12.0 \pm 4.7$ & $622.85623 \pm 0.00016$ & $0.0863 \pm 0.0074$ \\
\hline J210308.79-002748.9 & $0.20308 \pm 0.00023$ & $281.0 \pm 3.2$ & $-67.6 \pm 5.3$ & $384.87278 \pm 0.00474$ & $0.4666 \pm 0.0159$ \\
\hline
\end{tabular}

this system implies that the companion is almost certainly of low mass, regardless of the exact period. For the best-fit orbital period, the companion is an $M \geqslant 0.32 M_{\odot}$ compact object. The probability of a 1.4-3.0 $M_{\odot}$ neutron star companion is only $6 \%$. Assuming the mean inclination angle for a random stellar sample, $i=60^{\circ}$, the companion is likely another low-mass WD with $M=0.41 M_{\odot}$. This binary will not merge within a Hubble time.

\section{2. $J 0825+1152$}

J0825+1152 has a well-constrained orbital period of 83.79 \pm 0.01 minutes. Because our eight-minute-long exposures span $10 \%$ of its orbital phase, the observed amplitude is underestimated by a factor of 0.985 . The corrected radial velocity semi-amplitude is $K=319.4 \mathrm{~km} \mathrm{~s}^{-1}$.

$\mathrm{J} 0825+1152$ has $T_{\text {eff }}=24,830 \pm 740 \mathrm{~K}$ and $\log g=$ $6.61 \pm 0.04$. Based on the Panei et al. (2007) tracks, it is a 40 Myr old $0.26 M_{\odot}$ WD at $1.6 \mathrm{kpc}$. Using the corrected orbital parameters, there is a $9 \%$ probability that the companion is a neutron star. For $i=60^{\circ}$, the most likely companion is a $0.61 M_{\odot} \mathrm{C} / \mathrm{O}$ WD. The likelihood that the system contains a pair of WDs whose total mass exceeds the Chandrasekhar mass is $4 \%$. The merger time due to gravitational wave radiation is less than 180 Myr.

\section{3. $J 0845+1624$}

The SDSS spectrum of J0845+1624 is best fit with a $T_{\text {eff }}=$ $17,430 \pm 640 \mathrm{~K}$ and $\log g=6.72 \pm 0.17$ model (Kleinman 2010), implying that it is an ELM WD. However, the SDSS spectrum is noisy for this relatively faint, $g_{0}=19.7 \mathrm{mag}$ (dereddened), object. Our MMT spectrum is best explained by a model with $T_{\text {eff }}=17,750 \pm 110 \mathrm{~K}$ and $\log g=7.42 \pm 0.08$. $\mathrm{J} 0845+1624$ is a 130 Myr old $0.40 \pm 0.02 M_{\odot}$ WD at $0.9 \mathrm{kpc}$.

Based on 24 different spectra, the best-fit orbital period is $18.1 \mathrm{hr}$ with $K=62.2 \pm 5.4 \mathrm{~km} \mathrm{~s}^{-1}$, but there are several significant aliases (e.g., at $10.4 \mathrm{hr}$ ). Given the relatively lowamplitude velocity variations, the orbital period is not well constrained. J0845+1624 has a companion more massive than $0.19 M_{\odot}$. The probability of a neutron star companion is only $2 \%$; the companion is most likely another low-mass WD. This binary will not merge within a Hubble time.

\section{4. $J 1005+0542$}

$\mathrm{J} 1005+0542$ was classified as an ELM WD with $T_{\text {eff }}=$ $15,190 \pm 490 \mathrm{~K}$ and $\log g=6.87 \pm 0.14$ based on its SDSS spectrum (Kleinman 2010). Our higher resolution and higher signal-to-noise ratio MMT spectrum of this $g_{0}=19.7 \mathrm{mag}$ object is best explained by a model with $T_{\text {eff }}=15,740 \pm 330 \mathrm{~K}$ and $\log g=7.25 \pm 0.06$. Hence, J1005+0542 is a $140 \mathrm{Myr}$ old $0.34 M_{\odot} \mathrm{WD}$ at $1 \mathrm{kpc}$.
The best-fit orbital period for $\mathrm{J} 1005+0542$ is $7.334 \pm 0.002 \mathrm{hr}$. The invisible companion is an $M \geqslant 0.66 M_{\odot}$ compact object. There is a $14 \%$ probability that the companion is a neutron star. Similarly, there is an $11 \%$ probability that the companion is a massive WD and the combined mass of the two stars is more than 1.4 $M_{\odot}$. For an average inclination angle of $i=60^{\circ}$, the companion is a $0.86 M_{\odot} \mathrm{WD}$.

\section{5. $J 1005+3550$}

$\mathrm{J} 1005+3550$ is only $4^{\prime \prime}$ away from a 15 th mag star. To avoid contamination from this nearby source, we kept the slit at a fixed orientation throughout the observations. We derive $T_{\text {eff }}=10,010 \pm 430 \mathrm{~K}$ and $\log g=5.82 \pm 0.11$ from the MMT composite spectrum. $\mathrm{J} 1005+3550$ is a $0.17 M_{\odot}$ WD with an absolute magnitude of $M_{g} \simeq 8$ and $d=1.5 \mathrm{kpc}$. J1005+3550 has a remarkable systemic velocity of $-171 \mathrm{~km} \mathrm{~s}^{-1}$, indicating a halo origin. Unfortunately, no proper motion measurements are available in the SDSS + USNO-B catalog (Munn et al. 2004).

The best-fit orbital period for $\mathrm{J} 1005+3550$ is $4.2 \mathrm{hr}$, but there are a few significant aliases (e.g., at $3.6 \mathrm{hr}$ ). In either case, $\mathrm{J} 1005+3550$ has a merger time shorter than a Hubble time. For the best-fit orbital period of $4.2 \mathrm{hr}$ and $K=143 \mathrm{~km} \mathrm{~s}^{-1}$, $\mathrm{J} 1005+3550$ has a relatively low-mass companion with $M \geqslant$ $0.19 M_{\odot}$. For an average inclination angle of $60^{\circ}$, the companion is a $0.24 M_{\odot} \mathrm{WD}$.

\section{6. $J 1056+6536$}

$\mathrm{J} 1056+6536$ is the shortest period system among the seven new systems discussed in this paper. It has a well-constrained period of $62.7 \pm 1.5$ minutes, making it the fifth-shortest period detached WD system currently known. Because our eight-minute-long exposures span $13 \%$ of its orbital phase, the observed amplitude is underestimated by a factor of 0.964 . The corrected radial velocity semi-amplitude is $K=267.5 \mathrm{~km} \mathrm{~s}^{-1}$.

$\mathrm{J} 1056+6536$ was originally classified as a $T_{\text {eff }}=21,910 \pm$ $1900 \mathrm{~K}$ and $\log g=7.07 \pm 0.10$ low-mass WD by Liebert et al. (2004) based on an SDSS spectrum. Eisenstein et al. (2006) analyze the same spectrum, and find a best-fit model with $T_{\text {eff }}=20,110 \pm 630 \mathrm{~K}$ and $\log g=6.94 \pm 0.12$. Our higher quality MMT spectrum is best explained by a model with $T_{\text {eff }}=20,470 \pm 520 \mathrm{~K}$ and $\log g=7.13 \pm 0.02$. This solution is consistent with the previous estimates within the errors. J1056+6536 is therefore a $50 \mathrm{Myr}$ old $0.34 M_{\odot} \mathrm{WD}$ at $1.4 \mathrm{kpc}$.

Based on the mass function, the companion is a $\geqslant 0.34 M_{\odot}$ compact object. No MSP companion is detected in the radio data (Agüeros et al. 2009a). Hence, the companion is almost certainly another WD. For an average inclination of $60^{\circ}$, it is a $0.43 M_{\odot}$ low-mass WD. The merger time due to gravitational wave radiation is $\leqslant 85 \mathrm{Myr}$. 


\subsection{J2103-0027}

J2103-0027 has $T_{\text {eff }}=10,000 \pm 300 \mathrm{~K}$ and $\log g=$ $5.49 \pm 0.10$. Like J1005+3550, we assign $M=0.17 M_{\odot}$ and an absolute magnitude of $M_{g} \simeq 8$, which corresponds to a distance of $1.1 \mathrm{kpc}$. J2103-0027 has a systemic velocity of $-67.6 \mathrm{~km} \mathrm{~s}^{-1}$ and a proper motion of $9.3 \pm 4.9{\text { mas } \mathrm{yr}^{-1}}^{-1}$ (Munn et al. 2004). Its kinematics are consistent with the disk population.

J2103-0027 has a well-constrained period of $4.874 \pm$ $0.006 \mathrm{hr}$. We observe peak-to-peak radial velocity variations of $562 \mathrm{~km} \mathrm{~s}^{-1}$. Hence, the companion is a relatively massive compact object with $M \geqslant 0.71 M_{\odot}$. There is a $17 \%$ probability that the companion is a neutron star. For $i=60^{\circ}$, the companion is a $0.99 M_{\odot} \mathrm{WD}$.

J2103-0027 displays a Ca K line in absorption with a $0.3 \AA$ equivalent width. So far, all known ELM WDs with $\log g<6$ display the Ca K line in absorption (Kilic et al. 2007; Brown et al. 2010, 2012; Vennes et al. 2011). The origin of $\mathrm{Ca}$ is unclear, but it is most likely related to accretion from the immediate circumstellar environment (see the discussion in Kilic et al. 2007; Vennes et al. 2011).

\section{DISCUSSION}

\subsection{Seven New Binary WD Systems}

We identify seven new detached short-period WD binary systems in the SDSS Data Release 7 area. All seven targets were classified as $\log g<7$ WDs based on SDSS spectroscopy (Eisenstein et al. 2006; Kleinman 2010). However, our MMT data show that three of the targets have $\log g>7$ and $M=$ 0.3-0.4 $M_{\odot}$. Assuming the mean inclination angle for a random stellar sample, $i=60^{\circ}$, the companions range from $0.22 M_{\odot}$ to $0.99 M_{\odot}$. The probability of neutron star companions is $\leqslant 17 \%$ for all systems. Hence, the companions are most likely other WDs. Radio observations are available only for one of these targets, J1056+6536, which is also the shortest period system in the current sample. There is no evidence of a pulsar companion in the radio data for $\mathrm{J} 1056+6536$.

Five of these targets have merger times $\leqslant 10$ Gyr. The fastest merger systems are J0825+1152 and J1056+6536. With $\tau_{\text {merge }}=85 \mathrm{Myr}, \mathrm{J} 1056+6536$ is currently the fifth-fastest merger system known.

There are six targets with SDSS and/or USNO-B proper motion measurements (Munn et al. 2004). However, none of them show significant proper motions, and these six systems have disk kinematics. The only star without a proper motion measurement, J1005+3550, has a systemic velocity indicative of halo objects, $|\gamma|=171 \mathrm{~km} \mathrm{~s}^{-1}$.

\subsection{The ELM WD Sample}

The ELM Survey has so far observed 40 low-mass WDs for radial velocity variations, discovering velocity variability in all but 4 of the targets. Two objects, J0651 and NLTT 11748 , are eclipsing double WD systems. No main-sequence companions are visible in the available optical photometry and spectroscopy data for the remaining 38 targets. There are a few cases where the probability of a neutron star companion is more than 10\%, like the intriguing system J1741 (Brown et al. 2012; Hermes et al. 2012). Radio or X-ray data are available for 14 systems (including the two sources presented in Section 2.2; van Leeuwen et al. 2007; Agüeros et al. 2009a, 2009b; Kilic et al.

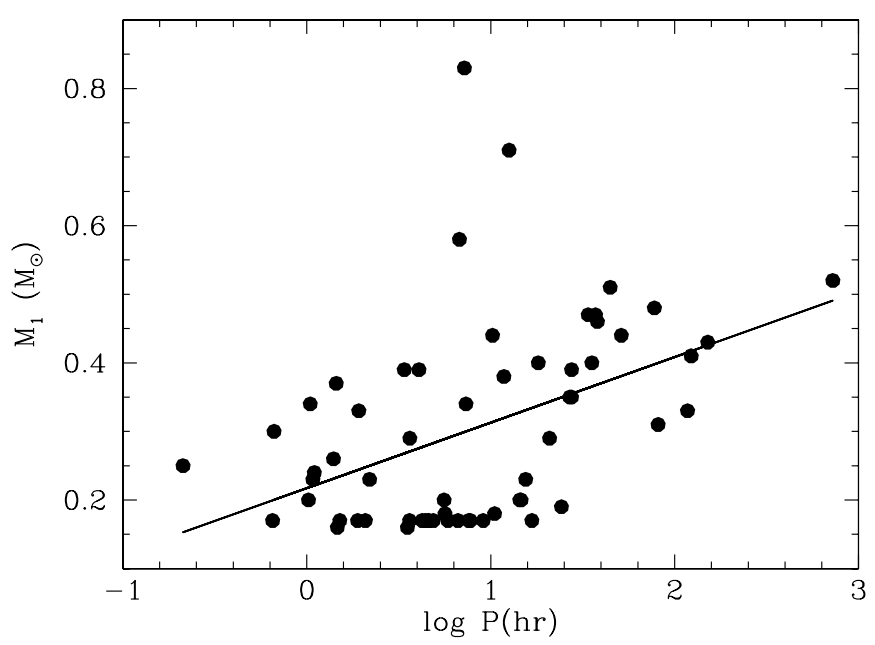

Figure 4. Population of observed double WDs (this paper and Nelemans et al. 2005) as a function of orbital period and mass of the brighter WD of the pair. The solid line is a least-squares fit to the data.

2011a). No MSP companions are detected in these data. Thus, the companions are most likely WDs.

\subsubsection{The Period Distribution of Binary WDs}

The orbital and physical parameters for the current merger and non-merger samples found in the ELM Survey are presented in Table 4. The orbital periods range from 12 minutes to 1.01 days with the median at $4.4 \mathrm{hr}$. This is significantly shorter than the median periods of $21 \mathrm{hr}$ and $6.7 \mathrm{hr}$ for more massive WDs (Nelemans et al. 2005) and for main sequence + He-core WD binaries (Zorotovic et al. 2011), respectively.

Figure 4 shows the masses and orbital periods for our sample of ELM WDs and the previously known double WD systems in the literature (Nelemans et al. 2005). Similar to the trend seen in WD + main-sequence post-common-envelope binaries (Zorotovic et al. 2011), lower mass WDs are found in shorter period systems. This figure demonstrates that the closest binary systems end up as ELM WDs. This is expected; shorter period systems would start interacting earlier in their evolution compared to longer period systems and experience enhanced mass loss during the red giant phase, and hence end up as lower mass WDs. There are now three ELM WD binaries known with $P<1 \mathrm{hr}$ and nine with $P=1-2 \mathrm{hr}$. The ELM Survey has discovered the eight shortest period double WDs currently known.

We have now observed 33 WDs with $M \leqslant 0.3 M_{\odot}$, of which 31 are velocity variable. The average velocity semiamplitude of these binaries is $266 \mathrm{~km} \mathrm{~s}^{-1}$, whereas the upper limits for the velocity semi-amplitudes of J0900+0234 $4^{11}$ and $\mathrm{J} 1448+1342$ are 24 and $35 \mathrm{~km} \mathrm{~s}^{-1}$, respectively. An average system viewed at $i \leqslant 7.6$ would be consistent with the observations of these two velocity non-variable systems. For a randomly distributed sample of orbital inclinations, there is a $0.9 \%$ chance that $i \leqslant 7.6$. Thus, there is a $30(9) \%$ likelihood of finding one(two) non-variable systems in a sample of 33 stars. It is possible that both $\mathrm{J} 0900+0234$ and $\mathrm{J} 1448+1342$ are poleon or long-period binary systems. Hence, the binary fraction of $M \leqslant 0.3 M_{\odot}$ WDs is at least $94 \%$ and it may be as high as $100 \%$.

\footnotetext{
$\overline{11}$ Brown et al. (2012) do not rule out binarity for J0900+0234.
} 
Table 4

Merger and Non-merger Systems in the ELM Survey

\begin{tabular}{|c|c|c|c|c|c|c|c|c|c|}
\hline Object & $\begin{array}{l}T_{\text {eff }} \\
(\mathrm{K})\end{array}$ & $\log g$ & $\begin{array}{c}P \\
\text { (days) }\end{array}$ & $\begin{array}{c}K \\
\left(\mathrm{~km} \mathrm{~s}^{-1}\right)\end{array}$ & $\begin{array}{l}\text { Mass } \\
\left(M_{\odot}\right)\end{array}$ & $\begin{array}{c}M_{2} \\
\left(M_{\odot}\right)\end{array}$ & $\begin{array}{c}M_{2}\left(60^{\circ}\right) \\
\left(M_{\odot}\right)\end{array}$ & $\begin{array}{l}\tau_{\text {merge }} \\
(\mathrm{Gyr})\end{array}$ & Reference \\
\hline J0022-1014 & 18980 & 7.15 & 0.07989 & 145.6 & 0.33 & $\geqslant 0.19$ & 0.23 & $\leqslant 0.73$ & 6 \\
\hline J0106-1000 & 16490 & 6.01 & 0.02715 & 395.2 & 0.17 & 0.43 & $\ldots$ & 0.037 & 7 \\
\hline J0112+1835 & 9690 & 5.63 & 0.14698 & 295.3 & 0.16 & $\geqslant 0.62$ & 0.85 & $\leqslant 2.7$ & 1 \\
\hline $\mathrm{J} 0651+2844$ & 16400 & 6.79 & 0.00885 & 657.3 & 0.25 & 0.55 & $\ldots$ & 0.0009 & 3 \\
\hline J0755+4906 & 13160 & 5.84 & 0.06302 & 438.0 & 0.17 & $\geqslant 0.81$ & 1.12 & $\leqslant 0.22$ & 2 \\
\hline $\mathrm{J} 0818+3536$ & 10620 & 5.69 & 0.18315 & 170.0 & 0.17 & $\geqslant 0.26$ & 0.33 & $\leqslant 8.9$ & 2 \\
\hline $\mathrm{J} 0822+2753$ & 8880 & 6.44 & 0.24400 & 271.1 & 0.17 & $\geqslant 0.76$ & 1.05 & $\leqslant 8.4$ & 4 \\
\hline $\mathrm{J} 0825+1152$ & 24830 & 6.61 & 0.05819 & 319.4 & 0.26 & $\geqslant 0.47$ & 0.61 & $\leqslant 0.18$ & 0 \\
\hline $\mathrm{J} 0849+0445$ & 10290 & 6.23 & 0.07870 & 366.9 & 0.17 & $\geqslant 0.64$ & 0.88 & $\leqslant 0.47$ & 4 \\
\hline $\mathrm{J} 0923+3028$ & 18350 & 6.63 & 0.04495 & 296.0 & 0.23 & $\geqslant 0.34$ & 0.44 & $\leqslant 0.13$ & 2 \\
\hline $\mathrm{J} 1005+0542$ & 15740 & 7.25 & 0.30560 & 208.9 & 0.34 & $\geqslant 0.66$ & 0.86 & $\leqslant 9.0$ & 0 \\
\hline $\mathrm{J} 1005+3550$ & 10010 & 5.82 & 0.17652 & 143.0 & 0.17 & $\geqslant 0.19$ & 0.24 & $\leqslant 10.3$ & 0 \\
\hline $\mathrm{J} 1053+5200$ & 15180 & 6.55 & 0.04256 & 264.0 & 0.20 & $\geqslant 0.26$ & 0.33 & $\leqslant 0.16$ & 4,9 \\
\hline $\mathrm{J} 1056+6536$ & 20470 & 7.13 & 0.04351 & 267.5 & 0.34 & $\geqslant 0.34$ & 0.43 & $\leqslant 0.085$ & 0 \\
\hline $\mathrm{J} 1233+1602$ & 10920 & 5.12 & 0.15090 & 336.0 & 0.17 & $\geqslant 0.86$ & 1.20 & $\leqslant 2.1$ & 2 \\
\hline $\mathrm{J} 1234-0228$ & 18000 & 6.64 & 0.09143 & 94.0 & 0.23 & $\geqslant 0.09$ & 0.11 & $\leqslant 2.7$ & 6 \\
\hline $\mathrm{J} 1436+5010$ & 16550 & 6.69 & 0.04580 & 347.4 & 0.24 & $\geqslant 0.46$ & 0.60 & $\leqslant 0.10$ & 4,9 \\
\hline $\mathrm{J} 1443+1509$ & 8810 & 6.32 & 0.19053 & 306.7 & 0.17 & $\geqslant 0.83$ & 1.15 & $\leqslant 4.1$ & 1 \\
\hline $\mathrm{J} 1630+4233$ & 14670 & 7.05 & 0.02766 & 295.9 & 0.30 & $\geqslant 0.30$ & 0.37 & $\leqslant 0.031$ & 8 \\
\hline $\mathrm{J} 1741+6526$ & 9790 & 5.19 & 0.06111 & 508.0 & 0.16 & $\geqslant 1.10$ & 1.55 & $\leqslant 0.17$ & 1 \\
\hline $\mathrm{J} 1840+6423$ & 9140 & 6.16 & 0.19130 & 272.0 & 0.17 & $\geqslant 0.64$ & 0.88 & $\leqslant 5.0$ & 1 \\
\hline $\mathrm{J} 2103-0027$ & 10000 & 5.49 & 0.20308 & 281.0 & 0.17 & $\geqslant 0.71$ & 0.99 & $\leqslant 5.4$ & 0 \\
\hline $\mathrm{J} 2119-0018$ & 10360 & 5.36 & 0.08677 & 383.0 & 0.17 & $\geqslant 0.75$ & 1.04 & $\leqslant 0.54$ & 2 \\
\hline NLTT 11748 & 8690 & 6.54 & 0.23503 & 273.4 & 0.18 & 0.76 & $\ldots$ & 7.2 & $5,10,11$ \\
\hline $\mathrm{J} 0022+0031$ & 17890 & 7.38 & 0.49135 & 80.8 & 0.38 & $\geqslant 0.21$ & 0.26 & $\ldots$ & 6 \\
\hline $\mathrm{J} 0152+0749$ & 10840 & 5.80 & 0.32288 & 217.0 & 0.17 & $\geqslant 0.57$ & 0.78 & $\ldots$ & 1 \\
\hline $\mathrm{J} 0730+1703$ & 11080 & 6.36 & 0.69770 & 122.8 & 0.17 & $\geqslant 0.32$ & 0.41 & $\ldots$ & 0 \\
\hline $\mathrm{J} 0845+1624$ & 17750 & 7.42 & 0.75599 & 62.2 & 0.40 & $\geqslant 0.19$ & 0.22 & $\ldots$ & 0 \\
\hline $\mathrm{J} 0900+0234$ & 8220 & 5.78 & $\ldots$ & $\leqslant 24$ & 0.16 & $\ldots$ & $\ldots$ & $\ldots$ & 1 \\
\hline $\mathrm{J} 0917+4638$ & 11850 & 5.55 & 0.31642 & 148.8 & 0.17 & $\geqslant 0.28$ & 0.36 & $\ldots$ & 12 \\
\hline $\mathrm{J} 1422+4352$ & 12690 & 5.91 & 0.37930 & 176.0 & 0.17 & $\geqslant 0.41$ & 0.55 & $\ldots$ & 2 \\
\hline $\mathrm{J} 1439+1002$ & 14340 & 6.20 & 0.43741 & 174.0 & 0.18 & $\geqslant 0.46$ & 0.62 & $\ldots$ & 2 \\
\hline $\mathrm{J} 1448+1342$ & 12580 & 6.91 & $\ldots$ & $\leqslant 35$ & 0.25 & $\ldots$ & $\ldots$ & $\ldots$ & 2 \\
\hline $\mathrm{J} 1512+2615$ & 12130 & 6.62 & 0.59999 & 115.0 & 0.20 & $\geqslant 0.28$ & 0.36 & $\ldots$ & 2 \\
\hline $\mathrm{J} 1518+0658$ & 9810 & 6.66 & 0.60935 & 172.0 & 0.20 & $\geqslant 0.58$ & 0.78 & $\ldots$ & 1 \\
\hline $\mathrm{J} 1625+3632$ & 23570 & 6.12 & 0.23238 & 58.4 & 0.20 & $\geqslant 0.07$ & 0.08 & $\ldots$ & 6 \\
\hline $\mathrm{J} 1630+2712$ & 11200 & 5.95 & 0.27646 & 218.0 & 0.17 & $\geqslant 0.52$ & 0.70 & $\ldots$ & 2 \\
\hline $\mathrm{J} 2252-0056$ & 19450 & 7.00 & $\ldots$ & $\leqslant 25$ & 0.31 & $\ldots$ & $\ldots$ & $\ldots$ & 2 \\
\hline $\mathrm{J} 2345-0102$ & 33130 & 7.20 & $\ldots$ & $\leqslant 43$ & 0.42 & $\ldots$ & $\ldots$ & $\ldots$ & 2 \\
\hline LP400-22 & 11170 & 6.35 & 1.01016 & 119.9 & 0.19 & $\geqslant 0.41$ & 0.52 & $\ldots$ & 13,14 \\
\hline
\end{tabular}

References. (0) This paper; (1) Brown et al. 2012; (2) Brown et al. 2010; (3) Brown et al. 2011c; (4) Kilic et al. 2010b; (5) Kilic et al. 2010a; (6) Kilic et al. 2011a; (7) Kilic et al. 2011c; (8) Kilic et al. 2011b; (9) Mullally et al. 2009; (10) Steinfadt et al. 2010; (11) Kawka et al. 2010; (12) Kilic et al. 2007; (13) Kilic et al. 2009; (14) Vennes et al. 2009.

\subsubsection{Two Dozen Merger Systems}

The physical parameters and space density of double degenerate merger systems are important for understanding the formation of R Coronae Borealis stars, single subdwarfs, AM CVn systems, and supernovae Ia and .Ia. However, previous surveys were unsuccessful in finding a large merger population. Figure 5 presents the total masses and periods for short-period double WD systems found in the literature (Nelemans et al. 2005; Napiwotzki et al. 2007); there were only six systems known to have short enough orbital periods to merge within a Hubble time. We discovered 24 merger systems in a sample of 40 stars observed, a success rate of $60 \%$. We have now quintupled the number of merger systems known, and we anticipate finding many more.

There are three more short-period binary WD systems discovered in recent years: SDSS J1005+2249 (Parsons et al.
2011), SDSS J1257+5428 (Badenes et al. 2009; Kulkarni \& van Kerkwijk 2010; Marsh et al. 2011), and GALEX J1717+6757 (Vennes et al. 2011). Not surprisingly, all three systems involve low-mass WD primaries.

Kilic et al. (2011a) compare the observed period and mass distribution of double WD systems with the population synthesis models of Nelemans et al. (2001b). Based on the population synthesis calculations, Kilic et al. (2011a) argue that there should not be many systems with periods less than an hour. The shortest period system known at that time was J1053+5200 with an orbital period of 61 minutes. Recent discoveries of three systems with 12-39 minute orbital periods indicate that $P<1 \mathrm{hr}$ detached binary WDs indeed exist. Unfortunately, the overall number distribution of the population synthesis models and our observations cannot be directly compared due to the complicated target selection biases in the SDSS. Nevertheless, the period distribution of our sample is informative. For example, 


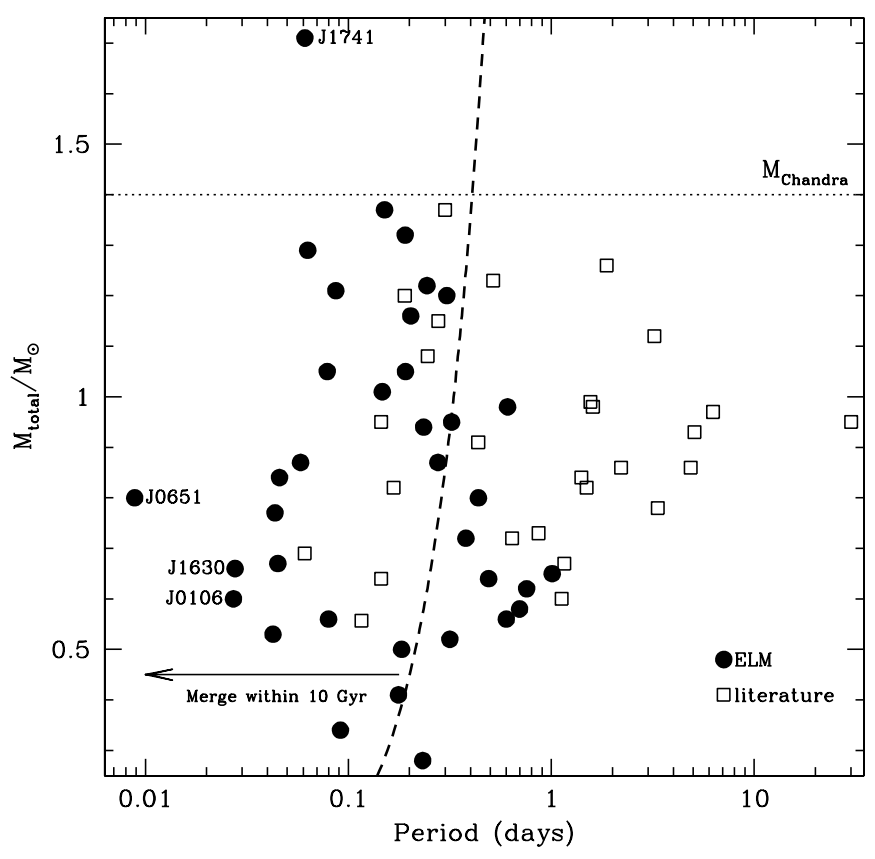

Figure 5. Binary orbital period vs. total system mass for the full ELM Survey and for the previously identified double WD systems (Nelemans et al. 2005; Napiwotzki et al. 2007; Marsh et al. 2011; Parsons et al. 2011; Vennes et al. 2011). We plot the total system mass assuming $i=60^{\circ}$ when the orbital inclination is unknown, and the correct system mass when the inclination is known either from eclipses or ellipsoidal variations. The dashed line shows the approximate threshold at which 1:1 mass ratio systems will merge in less than 10 Gyr.

depending on the WD cooling models used, the population synthesis calculations change significantly (Nelemans et al. 2001b). The relative number distribution of short-period systems in our survey can be used to constrain the population synthesis models.

Binary WDs provide important constraints on the commonenvelope phase, an evolutionary stage that is difficult to study because of its brevity. Nelemans et al. (2005) argue that the standard common-envelope ( $\alpha$-) formalism, equating the energy balance in the system, does not always work. Instead, they suggest that the common-envelope evolution of close WD binaries can be reconstructed with the $\gamma$-algorithm imposing angular momentum balance. They use the observed mass ratio distribution of double WDs $(q \sim 1)$ to demonstrate that the $\gamma$ mechanism with a single value can explain all of the known systems. Studying the prior evolution of two ELM WD binaries, Kilic et al. (2007) and Kilic et al. (2009) argue that the same is not true for ELM WDs (also see Woods et al. 2012). We now know that there are many ELM WDs with extreme mass ratios. The mass distribution of our sample of ELM WDs will be extremely useful for constraining the $\gamma$ and $\alpha$ mechanisms, although with the caveat that modeling two common envelope phases for our systems clearly has large uncertainties. De Marco et al. (2011) use the observed population of post-common-envelope binaries including the central stars of planetary nebulae to demonstrate that systems with small mass-ratios have higher values of $\alpha$. Understanding the prior evolution of the ELM WDs may benefit from a similar study.

\subsection{The Future: Merger Products}

The future evolution of the ELM WD systems depends on the mass ratio of the two components. Marsh et al. (2004) demonstrate that systems with extreme mass ratios of $q \ll 1$ will

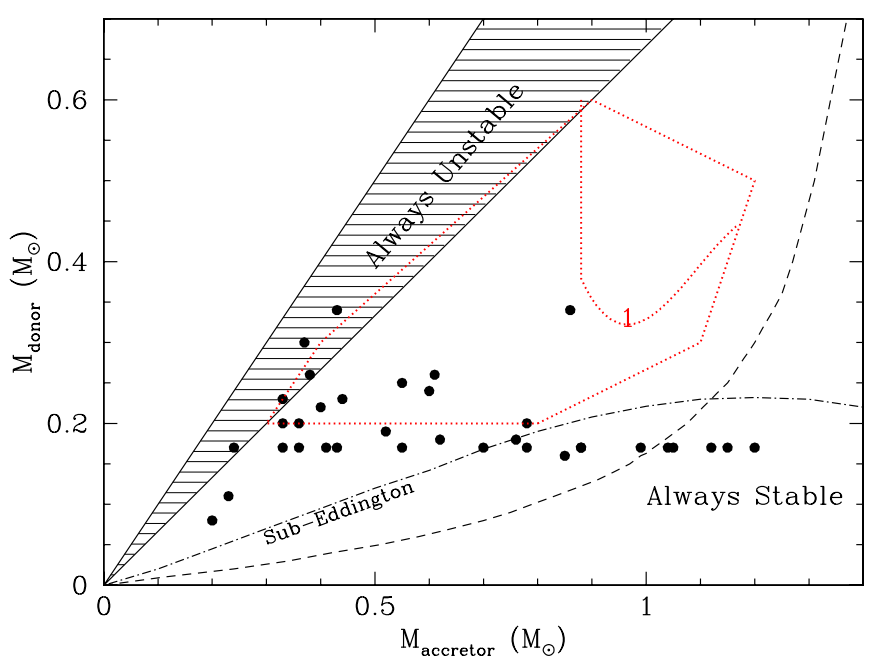

Figure 6. Mass transfer stability in double WD binaries (Marsh et al. 2004; Dan et al. 2011). Binary WDs (assuming $i=60^{\circ}$ for the systems with unknown inclinations) in the ELM Survey are shown as filled dots. Disk accretion occurs in the region below the dashed line. These systems will evolve into stable masstransfer AM CVn. The dot-dashed line separates sub- and super-Eddington accretion systems. Objects with mass ratios $q=2 / 3-1$ (hatched region) will have unstable mass transfer and merge. The area between the solid and dashed lines corresponds to either stable or unstable mass transfer depending on the spin-orbit coupling. The dotted line marks the area studied by Dan et al. (2011). The labeled contour (M. Dan 2011, private communication) marks the region where the triple- $\alpha$ burning timescale is equal to the dynamical timescale.

(A color version of this figure is available in the online journal.)

form a disk around the heavier WD, have stable mass transfer, and turn into interacting AM CVn binaries. If the transferred mass directly impacts the accretor, it can destabilize the orbit and lead to a merger. Figure 6 shows the observed mass distribution of the known ELM WDs and the stability criteria for different binaries (adapted from Marsh et al. 2004; Dan et al. 2011).

The mass transfer is unstable for mass ratios $q=2 / 3-1$ and stable for $q \ll 1$ (below the dashed line). The region between the solid and dashed lines corresponds to either stable or unstable mass transfer depending on the spin-orbit coupling. The dotdashed line separates sub- and super-Eddington accretion, with the former leading to stable mass transfer. Dan et al. (2011) perform simulations for a variety of primary and secondary masses including $0.2 M_{\odot}$ WDs (shown as dotted lines in Figure 6). All of the systems that they study, including a $0.2+$ $0.8 M_{\odot}$ binary WD system, have unstable mass transfer.

There are about a dozen ELM WDs that will have subEddington accretion rates and stable mass transfer. These systems are the progenitors of AM CVn and supernovae .Ia. There were no known progenitors of AM CVn systems before the ELM Survey. We have now identified about a dozen potential progenitors. Brown et al. (2011a) constrain the ELM WD space density using the WDs found in the magnitude-limited Hypervelocity star survey. They find that ELM WDs contribute at least a few percent to the AM CVn population. The subsequent discovery of the $<40$ minute orbital period systems indicates that the ELM WD contribution to the AM CVn population is a few times larger. However, the current sample of ELM WDs is not a complete sample due to the SDSS target selection biases. More accurate estimates for the ELM WD merger rate and space density have to wait until a larger magnitude-limited survey in a well-defined color range is completed (Brown et al. 2012).

The remaining targets in our sample will have superEddington accretion rates that would lead to unstable mass transfer and merger. There are at least five systems $(q=2 / 3-1)$ 


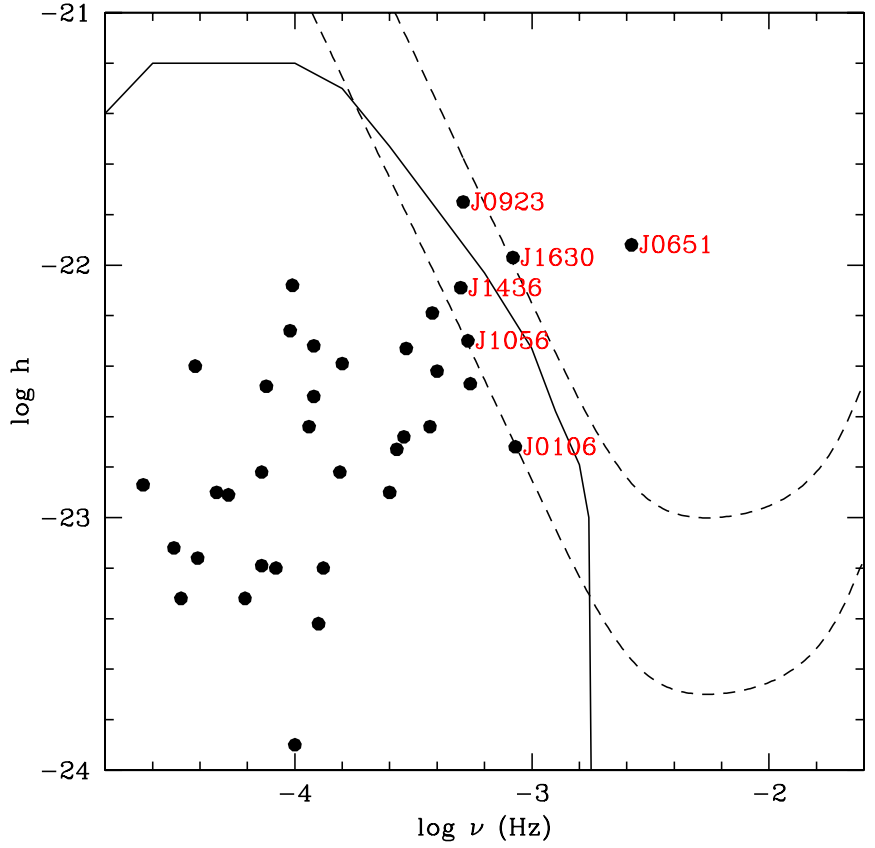

Figure 7. Predicted gravitational wave strain amplitudes $h$ and frequencies $v$ of the binary systems found in the ELM Survey. We assume an average inclination angle of $i=60^{\circ}$, except where the inclination is known from eclipses or ellipsoidal variations. The top and bottom dashed lines show the design sensitivities of LISA for a signal-to-noise ratio of 5 and 1, respectively, in one year of data collecting (Larson et al. 2000). The solid line shows the predicted Galactic foreground from Nelemans et al. (2001a). Sources above the $1 \sigma$ detection limit are labeled.

(A color version of this figure is available in the online journal.)

that should definitely have unstable mass transfer. Interestingly, the eight shortest-period systems will have super-Eddington accretion rates and end up as mergers. Depending on the unknown companion mass and composition, these systems are the progenitors of extreme helium stars, single subdwarfs, or massive WDs.

Guillochon et al. (2010) predict that Kelvin-Helmholtz instabilities in the accretion stream can lead to the detonation of a surface helium layer on a CO WD and perhaps the detonation of the WD itself, a potential mechanism to initiate a Type Ia supernova from binary WD mergers. Dan et al. (2011) show that the ratio of the triple- $\alpha$ burning timescale to the dynamical timescale is of the order of unity for the simulations that lead to a surface detonation. This ratio is $\gg 1$ for our targets (Figure 6). Hence, surface detonations through Kelvin-Helmholtz instabilities in the accretion stream are unlikely for our merging ELM WD targets.

\subsubsection{Gravitational Wave Sources}

Double degenerate binary WDs are important gravitational wave sources. Nelemans (2009) identifies 12 ultracompact binaries as LISA verification sources. There are eight AM CVn, three double degenerate, and one ultracompact X-ray binary sources currently known that should be detected by LISA with $\gtrsim 5 \sigma$ significance. Based on population synthesis calculations, Nelemans (2009) estimates that at least several hundred systems should be detectable by LISA.

Figure 7 shows the predicted gravitational wave strain amplitudes (Roelofs et al. 2007) and frequencies of the binary systems found in the ELM Survey. There are three binaries, J0651, J0923, and J1630, that should clearly be detected by a

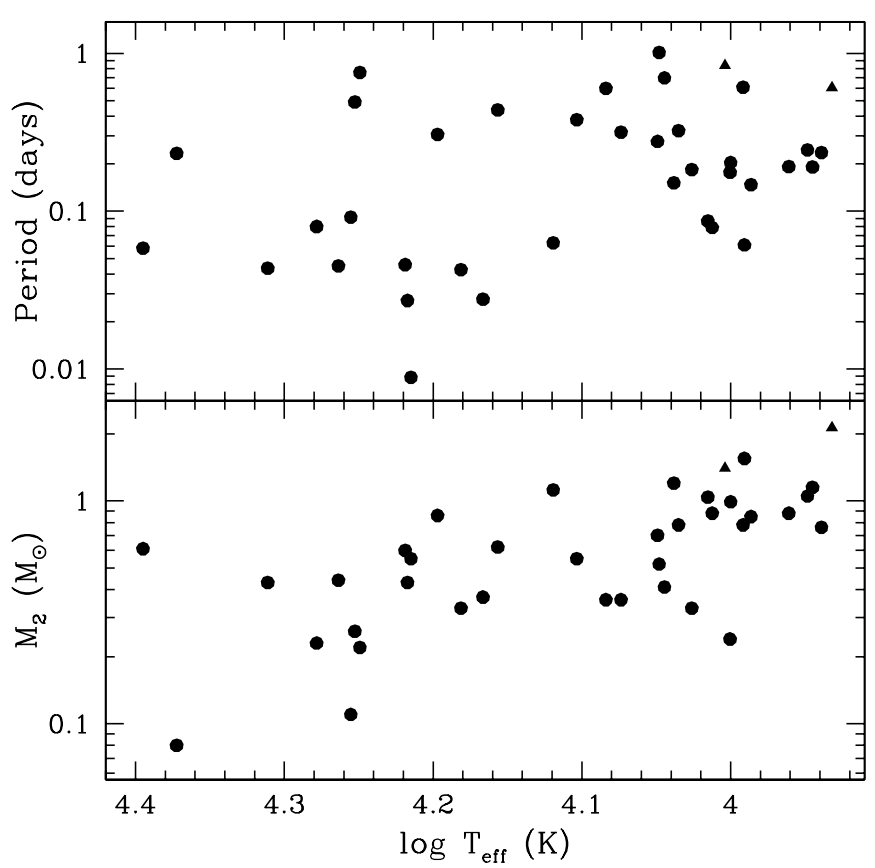

Figure 8. Periods and companion masses vs. temperatures for the 36 ELM WD binary systems in our sample (dots) plus the ELM WD companions to the MSPs PSR J1012+5307 and PSR J1911-5958A (triangles). We plot the companion masses assuming $i=60^{\circ}$ when the orbital inclination is unknown.

LISA-like mission within the first year of operation. This is a significant addition to the LISA verification sources. There are also three more systems that are above the $1 \sigma$ detection limit after one year of observations, but they may be lost in the Galactic foreground of unresolved double degenerate systems. However, it may be possible to identify these systems because we know their coordinates and physical parameters accurately from the optical observations. The remaining 30 sources are important indicators of what the Galactic foreground may look like for gravitational wave detectors.

\subsubsection{Trends}

With a sample of 36 binaries discovered in the ELM Survey, we can now search for observational trends. We have already mentioned the period differences between the ELM WD systems and the binary systems containing more massive WDs. So far, all ELM WDs that show radial velocity variations are in $\leqslant 1$ day orbits.

Using a sample of 19 merging ELM WD systems, Brown et al. (2012) show that there is an absence of cool ELM WDs with short orbital periods. Figure 8 presents periods versus temperatures for the 36 binary systems discussed here (see Table 4) and the ELM WD companions to two MSPs, PSR J1012+5307 (van Kerkwijk et al. 1996) and PSR J1911-5958A (Bassa et al. 2006). Clearly, the shortest-period systems are on average hotter than the longer-period systems. This period-temperature relation is likely because the shortest-period systems merge before they cool. A $0.2 M_{\odot}$ WD takes about $200 \mathrm{Myr}$ to cool down from $20,000 \mathrm{~K}$ to $10,000 \mathrm{~K}$ (Panei et al. 2007), whereas the merger times for $P<0.1$ day binaries are $\sim 100$ Myr.

Brown et al. (2012) note that there is also a correlation between the companion mass and the ELM WD's temperature. Such a trend is also observed for the 38 systems presented in Figure 8; hotter WDs tend to have less-massive companions. At first glance, there is no obvious reason for such a correlation. However, our interpretation is that the shortest-period systems 
Table 5

Radial Velocity Measurements

\begin{tabular}{|c|c|c|}
\hline Object & $\begin{array}{c}\text { HJD } \\
-2455000\end{array}$ & $\begin{array}{c}v_{\text {helio }} \\
\left(\mathrm{km} \mathrm{s}^{-1}\right)\end{array}$ \\
\hline J0730+1703 & 276.61626 & $181.3 \pm 19.7$ \\
\hline$\ldots$ & 276.62942 & $170.6 \pm 20.0$ \\
\hline$\ldots$ & 276.64002 & $222.1 \pm 12.5$ \\
\hline$\ldots$ & 276.71921 & $247.5 \pm 41.1$ \\
\hline$\ldots$ & 276.73155 & $229.0 \pm 37.7$ \\
\hline$\ldots$ & 276.74215 & $196.9 \pm 25.6$ \\
\hline$\ldots$ & 277.70070 & $19.2 \pm 40.2$ \\
\hline$\ldots$ & 277.70785 & $6.5 \pm 32.3$ \\
\hline$\ldots$ & 277.71731 & $-76.7 \pm 32.5$ \\
\hline$\ldots$ & 277.72444 & $32.7 \pm 34.2$ \\
\hline$\ldots$ & 277.73157 & $55.5 \pm 56.1$ \\
\hline$\ldots$ & 277.74074 & $-38.2 \pm 25.0$ \\
\hline$\ldots$ & 277.74787 & $-0.7 \pm 22.1$ \\
\hline$\ldots$ & 277.75503 & $-21.9 \pm 28.7$ \\
\hline$\ldots$ & 511.90070 & $197.5 \pm 14.4$ \\
\hline$\ldots$ & 512.90032 & $-36.2 \pm 16.8$ \\
\hline$\ldots$ & 531.84848 & $77.1 \pm 31.4$ \\
\hline$\ldots$ & 532.03716 & $205.2 \pm 27.1$ \\
\hline$\ldots$ & 532.85434 & $189.4 \pm 17.3$ \\
\hline$\ldots$ & 532.90336 & $143.7 \pm 21.9$ \\
\hline$\ldots$ & 533.85381 & $-82.8 \pm 32.8$ \\
\hline$\ldots$ & 533.88356 & $34.2 \pm 21.0$ \\
\hline$\ldots$ & 863.92955 & $228.2 \pm 18.3$ \\
\hline$\ldots$ & 864.92766 & $1.7 \pm 19.4$ \\
\hline$\ldots$ & 865.92156 & $244.4 \pm 20.1$ \\
\hline$\ldots$ & 866.98989 & $33.8 \pm 15.1$ \\
\hline J0825+1152 & 511.93759 & $353.2 \pm 18.4$ \\
\hline$\ldots$ & 512.91128 & $-52.6 \pm 22.2$ \\
\hline$\ldots$ & 531.85847 & $-123.4 \pm 16.0$ \\
\hline$\ldots$ & 531.91384 & $-54.4 \pm 13.8$ \\
\hline$\ldots$ & 531.92100 & $-218.1 \pm 8.7$ \\
\hline$\ldots$ & 531.94606 & $216.5 \pm 11.1$ \\
\hline$\ldots$ & 531.95182 & $354.8 \pm 10.5$ \\
\hline$\ldots$ & 531.95851 & $315.3 \pm 9.4$ \\
\hline$\ldots$ & 531.96428 & $202.1 \pm 12.9$ \\
\hline$\ldots$ & 531.97084 & $-18.3 \pm 8.6$ \\
\hline$\ldots$ & 531.97659 & $-191.9 \pm 10.8$ \\
\hline$\ldots$ & 531.98313 & $-265.2 \pm 12.0$ \\
\hline$\ldots$ & 531.98892 & $-242.8 \pm 12.2$ \\
\hline$\ldots$ & 531.99471 & $-102.6 \pm 13.5$ \\
\hline$\ldots$ & 532.04551 & $-296.6 \pm 24.4$ \\
\hline$\ldots$ & 533.03989 & $-177.6 \pm 10.9$ \\
\hline$\ldots$ & 533.87295 & $329.5 \pm 12.5$ \\
\hline J0845+1624 & 511.95127 & $49.5 \pm 52.7$ \\
\hline$\ldots$ & 512.92221 & $-26.8 \pm 33.7$ \\
\hline$\ldots$ & 532.96104 & $103.7 \pm 17.5$ \\
\hline$\ldots$ & 532.97245 & $57.5 \pm 16.0$ \\
\hline$\ldots$ & 532.98316 & $31.5 \pm 16.2$ \\
\hline$\ldots$ & 533.86319 & $88.9 \pm 12.4$ \\
\hline$\ldots$ & 533.89494 & $10.8 \pm 24.2$ \\
\hline$\ldots$ & 623.78330 & $45.0 \pm 22.1$ \\
\hline$\ldots$ & 674.63745 & $24.3 \pm 28.2$ \\
\hline$\ldots$ & 674.71316 & $-49.1 \pm 30.2$ \\
\hline$\ldots$ & 688.68475 & $68.1 \pm 32.1$ \\
\hline$\ldots$ & 689.64873 & $54.2 \pm 37.6$ \\
\hline$\ldots$ & 689.65468 & $120.3 \pm 38.6$ \\
\hline$\ldots$ & 689.67658 & $105.0 \pm 56.3$ \\
\hline$\ldots$ & 689.68299 & $57.2 \pm 48.0$ \\
\hline$\ldots$ & 690.67644 & $28.7 \pm 33.4$ \\
\hline$\ldots$ & 690.68216 & $-96.2 \pm 30.6$ \\
\hline$\ldots$ & 690.68934 & $-72.6 \pm 34.3$ \\
\hline$\ldots$ & 690.69542 & $-35.4 \pm 70.8$ \\
\hline$\ldots$ & 862.98606 & $-1.4 \pm 63.0$ \\
\hline$\ldots$ & 863.98615 & $17.6 \pm 19.2$ \\
\hline
\end{tabular}

Table 5

(Continued)

\begin{tabular}{|c|c|c|}
\hline Object & $\begin{array}{c}\text { HJD } \\
-2455000\end{array}$ & $\begin{array}{c}v_{\text {helio }} \\
\left(\mathrm{km} \mathrm{s}^{-1}\right)\end{array}$ \\
\hline$\ldots$ & 864.94448 & $72.9 \pm 24.8$ \\
\hline$\ldots$ & 865.94358 & $-57.9 \pm 36.9$ \\
\hline$\ldots$ & 866.99856 & $4.1 \pm 18.9$ \\
\hline $\mathrm{J} 1005+0542$ & 511.01107 & $85.8 \pm 27.9$ \\
\hline$\ldots$ & 512.95624 & $-186.5 \pm 37.0$ \\
\hline$\ldots$ & 532.99457 & $142.7 \pm 14.1$ \\
\hline$\ldots$ & 533.00541 & $104.4 \pm 16.4$ \\
\hline$\ldots$ & 533.01497 & $18.4 \pm 19.7$ \\
\hline$\ldots$ & 533.90538 & $169.0 \pm 15.6$ \\
\hline$\ldots$ & 533.99118 & $-144.5 \pm 20.8$ \\
\hline$\ldots$ & 534.00134 & $-192.4 \pm 24.2$ \\
\hline$\ldots$ & 534.01081 & $-178.6 \pm 18.4$ \\
\hline$\ldots$ & 534.02108 & $-173.4 \pm 40.5$ \\
\hline$\ldots$ & 534.02892 & $-251.8 \pm 28.7$ \\
\hline$\ldots$ & 623.85700 & $-173.6 \pm 28.1$ \\
\hline$\ldots$ & 674.65150 & $-112.3 \pm 30.8$ \\
\hline$\ldots$ & 675.63714 & $233.3 \pm 57.1$ \\
\hline$\ldots$ & 675.64309 & $196.8 \pm 21.1$ \\
\hline$\ldots$ & 688.69748 & $-132.8 \pm 39.4$ \\
\hline$\ldots$ & 863.00494 & $-77.0 \pm 29.0$ \\
\hline$\ldots$ & 863.99366 & $230.6 \pm 19.2$ \\
\hline$\ldots$ & 864.99933 & $55.5 \pm 28.6$ \\
\hline$\ldots$ & 865.98630 & $-253.7 \pm 24.3$ \\
\hline $\mathrm{J} 1005+3550$ & 533.03024 & $-198.2 \pm 19.0$ \\
\hline$\ldots$ & 533.04821 & $-180.6 \pm 14.3$ \\
\hline$\ldots$ & 533.92569 & $-285.7 \pm 26.5$ \\
\hline$\ldots$ & 533.93074 & $-155.7 \pm 52.4$ \\
\hline$\ldots$ & 533.93717 & $-126.6 \pm 27.6$ \\
\hline$\ldots$ & 533.94455 & $-110.7 \pm 8.9$ \\
\hline$\ldots$ & 533.95157 & $-74.1 \pm 7.0$ \\
\hline$\ldots$ & 533.95768 & $-52.8 \pm 7.6$ \\
\hline$\ldots$ & 533.96344 & $-55.0 \pm 7.0$ \\
\hline$\ldots$ & 533.97009 & $-31.0 \pm 8.1$ \\
\hline$\ldots$ & 533.97583 & $-17.1 \pm 6.6$ \\
\hline$\ldots$ & 533.98159 & $-26.6 \pm 6.0$ \\
\hline$\ldots$ & 534.04233 & $-275.0 \pm 16.5$ \\
\hline$\ldots$ & 534.04776 & $-296.7 \pm 13.3$ \\
\hline$\ldots$ & 674.66205 & $-107.2 \pm 33.8$ \\
\hline$\ldots$ & 674.66829 & $-51.6 \pm 18.6$ \\
\hline$\ldots$ & 674.72501 & $-256.6 \pm 6.5$ \\
\hline$\ldots$ & 674.73147 & $-273.9 \pm 9.6$ \\
\hline$\ldots$ & 675.69163 & $-114.9 \pm 23.8$ \\
\hline$\ldots$ & 675.71884 & $-65.9 \pm 16.3$ \\
\hline$\ldots$ & 676.68329 & $-292.5 \pm 29.2$ \\
\hline$\ldots$ & 689.75147 & $-324.6 \pm 9.5$ \\
\hline $\mathrm{J} 1056+6536$ & 622.85315 & $-149.9 \pm 15.8$ \\
\hline$\ldots$ & 674.74035 & $59.2 \pm 24.2$ \\
\hline$\ldots$ & 674.75039 & $-255.4 \pm 22.0$ \\
\hline$\ldots$ & 674.75893 & $-141.8 \pm 23.9$ \\
\hline$\ldots$ & 674.76777 & $164.4 \pm 28.4$ \\
\hline$\ldots$ & 674.77358 & $225.2 \pm 22.4$ \\
\hline$\ldots$ & 674.77943 & $256.7 \pm 35.8$ \\
\hline$\ldots$ & 674.78772 & $-89.6 \pm 27.4$ \\
\hline$\ldots$ & 676.78869 & $-216.8 \pm 48.7$ \\
\hline$\ldots$ & 676.79582 & $-241.5 \pm 59.9$ \\
\hline$\ldots$ & 688.77342 & $-12.0 \pm 14.5$ \\
\hline$\ldots$ & 688.77879 & $139.2 \pm 16.3$ \\
\hline$\ldots$ & 688.78617 & $228.2 \pm 44.2$ \\
\hline$\ldots$ & 688.79154 & $131.4 \pm 29.3$ \\
\hline $\mathrm{J} 2103-0027$ & 384.92248 & $200.9 \pm 10.9$ \\
\hline$\ldots$ & 384.93609 & $214.8 \pm 7.4$ \\
\hline$\ldots$ & 385.89359 & $-38.5 \pm 7.3$ \\
\hline$\ldots$ & 385.92301 & $168.1 \pm 13.5$ \\
\hline
\end{tabular}


Table 5

(Continued)

\begin{tabular}{lcc}
\hline \hline Object & $\begin{array}{c}\text { HJD } \\
-2455000\end{array}$ & $\begin{array}{c}v_{\text {helio }} \\
\left(\mathrm{km} \mathrm{s}^{-1}\right)\end{array}$ \\
\hline$\ldots$ & 387.91069 & $-153.7 \pm 9.2$ \\
$\ldots$ & 387.91855 & $48.3 \pm 11.7$ \\
$\ldots$ & 387.93947 & $85.2 \pm 7.4$ \\
$\ldots$ & 387.94731 & $152.7 \pm 9.9$ \\
$\ldots$ & 387.95648 & $165.2 \pm 12.5$ \\
$\ldots$ & 387.96676 & $250.5 \pm 10.9$ \\
$\ldots$ & 395.88891 & $213.2 \pm 42.6$ \\
$\ldots$ & 395.89969 & $199.1 \pm 7.4$ \\
$\ldots$ & 395.91083 & $130.1 \pm 13.1$ \\
$\ldots$ & 395.92039 & $95.9 \pm 14.8$ \\
$\ldots$ & 395.96147 & $-244.4 \pm 6.9$ \\
$\ldots$ & 395.97105 & $-294.8 \pm 9.2$ \\
$\ldots$ & 395.97876 & $-340.6 \pm 8.7$ \\
$\ldots$ & 396.90619 & $194.9 \pm 9.4$ \\
$\ldots$ & 396.91563 & $176.7 \pm 11.2$ \\
$\ldots$ & 396.92394 & $161.4 \pm 14.0$ \\
$\ldots$ & 396.95404 & $-19.8 \pm 8.8$ \\
$\ldots$ & 396.96331 & $-132.6 \pm 10.7$ \\
$\ldots$ & 531.61404 & $-204.9 \pm 29.1$ \\
\hline & &
\end{tabular}

start interacting earlier in their evolution, go through one or two common-envelope phases relatively quickly, and end up with lower mass companions. Since short-period systems also merge before they get a chance to cool down to $\sim 10,000 \mathrm{~K}$, there should be a deficit of cooler systems with lower mass companions. We only include ELM WD companions to two MSPs in Figure 8, but there are many MSPs with suspected ELM WD companions. In most cases, the companions are too faint for optical spectroscopy. The companions to the majority of these pulsars are $\sim 10$ Gyr old, and hence they should be 4000 K WDs (see Durant et al. 2012). Hence, there are many examples of cool WDs with massive (neutron star) companions.

\section{CONCLUSIONS}

We present seven new ELM WD binary systems, including five new merger systems. The shortest-period system in the new sample is $\mathrm{J} 1056+6536$, with an orbital period of 62.7 minutes; it will merge within $85 \mathrm{Myr}$ due to gravitational wave radiation. We have now identified 24 merging WD systems in the ELM Survey, quintupling the number of double WD systems known. We present an overview of the characteristics of this sample, including the period and mass distributions. Surface detonations due to instabilities in the accretion stream are unlikely for these systems. Hence, we expect merging ELM WD systems to evolve into stable mass-transfer AM CVn or unstable mass-transfer (merger) systems that would lead to the formation of single subdwarf, extreme helium star, or massive WDs. There are about a dozen ELM WD systems with extreme mass ratios that are potential progenitors of AM CVn systems and supernovae .Ia explosions.

We discuss the expected gravitational wave strain for our targets. There are six ELM WDs that may be detected by a LISA-like mission with a signal-to-noise ratio of $\geqslant 1$ after one year of observations. The remaining targets will be part of the Galactic foreground for gravitational wave detectors at low frequencies. We expect that our continuing observations will lead to more merging ELM WD discoveries, and improved constraints on the nature of their companions and their gravitational wave signal.
We thank M. Alegria, J. McAfee, A. Milone, and J. DiMiceli for their assistance with observations at the MMT Observatory. C.O.H. is supported by NSERC and an Ingenuity New Faculty Award. M.A.A. gratefully acknowledges Chandra grant GO1-12019X for support of portions of this program.

Facilities: MMT (Blue Channel Spectrograph), CXO (ACIS)

\section{APPENDIX \\ DATA TABLE}

Table 5 presents our radial velocity measurements. The table columns include object name, heliocentric Julian date, heliocentric radial velocity, and velocity error.

\section{REFERENCES}

Agüeros, M. A., Camilo, F., Silvestri, N. M., et al. 2009a, ApJ, 697, 283 Agüeros, M. A., Heinke, C., Camilo, F., et al. 2009b, ApJ, 700, L123 Allende Prieto, C., Beers, T. C., Wilhelm, R., et al. 2006, ApJ, 636, 804 Badenes, C., Mullally, F., Thompson, S. E., \& Lupton, R. H. 2009, ApJ, 707, 971

Bassa, C. G., van Kerkwijk, M. H., Koester, D., \& Verbunt, F. 2006, A\&A, 456, 295

Beloborodov, A. M. 2002, ApJ, 566, L85

Bildsten, L., Shen, K. J., Weinberg, N. N., \& Nelemans, G. 2007, ApJ, 662, L95 Bogdanov, S., Grindlay, J. E., \& Rybicki, G. B. 2006, ApJ, 648, L55

Brown, W. R., Geller, M. J., Kenyon, S. J., \& Kurtz, M. J. 2006, ApJ, 647, 303

Brown, W. R., Kilic, M., Allende Prieto, C., \& Kenyon, S. J. 2010, ApJ, 723, 1072

Brown, W. R., Kilic, M., Allende Prieto, C., \& Kenyon, S. J. 2011a, MNRAS, 411, L31

Brown, W. R., Kilic, M., Allende Prieto, C., \& Kenyon, S. J. 2012, ApJ, 744, 142

Brown, J. M., Kilic, M., Brown, W. R., \& Kenyon, S. J. 2011b, ApJ, 730, 67 Brown, W. R., Kilic, M., Hermes, J. J., et al. 2011c, ApJ, 737, L23

Dan, M., Rosswog, S., Guillochon, J., \& Ramirez-Ruiz, E. 2011, ApJ, 737, 89

De Marco, O., Passy, J.-C., Moe, M., et al. 2011, MNRAS, 411, 2277

Dickey, J. M., \& Lockman, F. J. 1990, ARA\&A, 28, 215

Durant, M., Kargaltsev, O., Pavlov, G. G., et al. 2012, ApJ, 746, 6

Eisenstein, D. J., Liebert, J., Harris, H. C., et al. 2006, ApJS, 167, 40

Guillochon, J., Dan, M., Ramirez-Ruiz, E., \& Rosswog, S. 2010, ApJ, 709, L64

Heinke, C. O., Grindlay, J. E., Edmonds, P. D., et al. 2005, ApJ, 625, 796

Hermes, J. J., Kilic, M., Brown, W. R., Montogemery, M. H., \& Winget, D. E. 2012, ApJ, 749, 42

Iben, I., Jr., \& Tutukov, A. V. 1984, ApJS, 54, 335

Kawka, A., Vennes, S., \& Vaccaro, T. R. 2010, A\&A, 516, L7

Kenyon, S. J., \& Garcia, M. R. 1986, AJ, 91, 125

Kilic, M., Allende Prieto, C., Brown, W. R., et al. 2010a, ApJ, 721, L158

Kilic, M., Brown, W. R., Allende Prieto, C., Kenyon, S. J., \& Panei, J. A. 2010b, ApJ, 716, 122

Kilic, M., Brown, W. R., Allende Prieto, C., Pinsonneault, M. H., \& Kenyon, S. J. 2007, ApJ, 664, 1088

Kilic, M., Brown, W. R., Allende Prieto, C., et al. 2009, ApJ, 695, L92

Kilic, M., Brown, W. R., Allende Prieto, C., et al. 2011a, ApJ, 727, 3

Kilic, M., Brown, W. R., Hermes, J. J., et al. 2011b, MNRAS, 418, L157

Kilic, M., Brown, W. R., Kenyon, S. J., et al. 2011c, MNRAS, 413, L101

Kleinman, S. J. 2010, in AIP Conf. Ser. 1273, 17th European White Dwarf Workshop, ed. K. Werner \& T. Rauch (Melville, NY: AIP), 156

Kroupa, P., \& Tout, C. A. 1997, MNRAS, 287, 402

Kulkarni, S. R., \& van Kerkwijk, M. H. 2010, ApJ, 719, 1123

Kurtz, M. J., \& Mink, D. J. 1998, PASP, 110, 934

Larson, S. L., Hiscock, W. A., \& Hellings, R. W. 2000, Phys. Rev. D, 62, 062001

Liebert, J., Bergeron, P., Eisenstein, D., et al. 2004, ApJ, 606, L147

Marsh, T. R., Dhillon, V. S., \& Duck, S. R. 1995, MNRAS, 275, 828

Marsh, T. R., Gänsicke, B. T., Steeghs, D., et al. 2011, ApJ, 736, 95

Marsh, T. R., Nelemans, G., \& Steeghs, D. 2004, MNRAS, 350, 113

Massey, P., Strobel, K., Barnes, J. V., \& Anderson, E. 1988, ApJ, 328, 315

Monet, D. G., Levine, S. E., Canzian, B., et al. 2003, AJ, 125, 984

Mullally, F., Badenes, C., Thompson, S. E., \& Lupton, R. 2009, ApJ, 707, L51

Munn, J. A., Monet, D. G., Levine, S. E., et al. 2004, AJ, 127, 3034

Napiwotzki, R., Edelmann, H., Heber, U., et al. 2001, A\&A, 378, L17

Napiwotzki, R., Karl, C. A., Nelemans, G., et al. 2007, in ASP Conf. Ser. 372, 15 th European Workshop on White Dwarfs, ed. R. Napiwotzki \& M. R. Burleigh (San Francisco, CA: ASP), 387 
Nelemans, G. 2009, Class. Quantum Gravity, 26, 094030

Nelemans, G., Napiwotzki, R., Karl, C., et al. 2005, A\&A, 440, 1087

Nelemans, G., Yungelson, L. R., \& Portegies Zwart, S. F. 2001a, A\&A, 375, 890

Nelemans, G., Yungelson, L. R., Portegies Zwart, S. F., \& Verbunt, F. 2001b, A\&A, 365, 491

Panei, J. A., Althaus, L. G., Chen, X., \& Han, Z. 2007, MNRAS, 382, 779

Parsons, S. G., Marsh, T. R., Gänsicke, B. T., Drake, A. J., \& Koester, D. 2011, ApJ, 735, L30

Roelofs, G. H. A., Groot, P. J., Benedict, G. F., et al. 2007, ApJ, 666, 1174

Serenelli, A. M., Althaus, L. G., Rohrmann, R. D., \& Benvenuto, O. G. 2001, MNRAS, 325, 607

Serenelli, A. M., Althaus, L. G., Rohrmann, R. D., \& Benvenuto, O. G. 2002, MNRAS, 337, 1091
Steinfadt, J. D. R., Kaplan, D. L., Shporer, A., Bildsten, L., \& Howell, S. B. 2010, ApJ, 716, L146

van Kerkwijk, M. H., Bergeron, P., \& Kulkarni, S. R. 1996, ApJ, 467, L89

van Leeuwen, J., Ferdman, R. D., Meyer, S., \& Stairs, I. 2007, MNRAS, 374, 1437

Vennes, S., Kawka, A., Vaccaro, T. R., \& Silvestri, N. M. 2009, A\&A, 507, 1613

Vennes, S., Thorstensen, J. R., Kawka, A., et al. 2011, ApJ, 737, L16

Voges, W., Aschenbach, B., Boller, Th., et al. 1999, A\&A, 349, 389

Webbink, R. F. 1984, ApJ, 277, 355

Woods, T. E., Ivanova, N., van der Sluys, M. V., \& Chaichenets, S. 2012, ApJ, 744,12

Zorotovic, M., Schreiber, M. R., Gänsicke, B. T., et al. 2011, A\&A, 536, L3 